\title{
Enhancer and super-enhancer landscape in polycystic kidney disease
}

Ronak Lakhia ${ }^{1 *}$, Abheepsa Mishra1*, Laurence Biggers, Venkat Malladi², Patricia Cobo-Stark ${ }^{1}$, Sachin Hajarnis ${ }^{1}$, and Vishal Patel ${ }^{1}$

${ }^{1}$ Department of Internal Medicine, Nephrology, UT Southwestern Medical Center, Dallas, Texas, USA 75390.

${ }^{2}$ Lyda Hill Department of Bioinformatics, UT Southwestern Medical Center, Dallas, TX

${ }^{*}$ co-first authors

Address correspondence: Ronak Lakhia, 5323 Harry Hines Boulevard, Dallas, Texas, USA

75390. Email: $\underline{\text { Ronak.Lakhia@utsouthwestern.edu }}$

Keywords: Polycystic kidney disease, enhancers, super-enhancers, H3K27ac, ChIP-Seq, c-Myc, Jun, Fos, epigenetics 


\begin{abstract}
Widespread aberrant gene expression is pathological hallmark of polycystic kidney disease (PKD). Numerous pathogenic signaling cascades, including c-Myc, Fos, and Jun are transactivated. However, the underlying epigenetic regulators are poorly defined. Here we show that H3K27ac, a histone modification that marks active enhancers, is elevated in mouse and human ADPKD samples. Using comparative H3K27ac ChIP-Seq analysis, we mapped >16000 active intronic and intergenic enhancer elements in Pkd1-mutant mouse kidneys. We find that the cystic kidney epigenetic landscape resembles that of a developing kidney, and $>90 \%$ of upregulated genes in Pkd1-mutant kidneys are co-housed with activated enhancers in the same topologically associated domains. Furthermore, we identify an evolutionarily-conserved enhancer cluster downstream of the $c-M y c$ gene and super-enhancers flanking both Jun and Fos loci in mouse and human ADPKD models. Deleting these regulatory elements reduces $c-M y c$, Jun, or Fos abundance and suppresses proliferation and 3D cyst growth of Pkd1-mutant cells. Finally, inhibiting glycolysis and glutaminolysis or activating Ppara in Pkd1-mutant cells lowers global $\mathrm{H} 3 \mathrm{~K} 27 \mathrm{ac}$ levels and on $c-M y c$ enhancers. Thus, our work suggests that epigenetic rewiring mediates the transcriptomic dysregulation in PKD, and the regulatory elements can be targeted to slow cyst growth.
\end{abstract}




\section{INTRODUCTION}

Autosomal Dominant Polycystic Kidney Disease (ADPKD), primarily caused by mutations in the PKD1 or PKD2 genes, affects nearly 12 million individuals worldwide and equally affects individuals irrespective of gender or race ${ }^{1}$. Fifty percent of individuals with ADPKD develop kidney failure requiring dialysis or kidney transplant ${ }^{2}$. The clinical hallmark of ADPKD is the massive bilateral kidney enlargement due to numerous kidney tubule-derived cysts. These cysts are lined by rapidly proliferating and metabolically deranged tubular epithelial cells ${ }^{3-7}$, fueling their progressive and relentless growth. Additionally, there is substantial interstitial inflammation and fibrosis, which also contribute to the decline in kidney function ${ }^{8}$. Tolvaptan, a vasopressin receptor 2 antagonist, slows the rate of kidney function decline and is the only FDA-approved treatment 9,10. There are other emerging therapeutic modalities ${ }^{11-14}$, but ADPKD pathogenesis is still incompletely understood, and there is a dire need for uncovering new drug targets.

Enhancers are dynamic cis-regulatory DNA elements (CREs), approximately 200-2,000 bp in length, that help shape cellular and tissue identity by fine-tuning transcriptomic makeup ${ }^{15}$. Active enhancers are marked by the transcriptionally-permissive H3K27 acetylation (H3K27ac) histone modification and accessible chromatin that allows the binding of sequence and cell typespecific transcription factors, transcriptional co-activators, and RNA polymerase II (RNAP II). A subset of CREs is referred to as super-enhancers ${ }^{16-18}$. These span large genomic regions (several kb in length) and exhibit unusually high transcriptional factor binding density and H3K27ac histone modifications. Active enhancers/super-enhancers physically interact with gene promoters, often over long genomic distances, to regulate gene transcription ${ }^{15}$. Unlike promoters, enhancers/super-enhancers can be present upstream or downstream of their target genes and are capable of activating gene transcription in any orientation. The enhancer/super-enhancer landscape is tissue-specific, contributing to each organ's unique transcriptomic output. Enhancers are also dynamically regulated, facilitating gene expression switches accompanying tissue 
developmental stage. With regards to diseases, enhancer biology is best studied in cancer, where many tumor-specific enhancers and super-enhancers have been reported. Moreover, targeting enhancers has shown early promise as a novel method to reduce pathogenic gene expression. However, barring a few exceptions ${ }^{19,20}$, enhancer impact on most kidney diseases still remains poorly defined.

Large-scale transcriptomic dysregulation is a pathological hallmark of ADPKD. Numerous pro-proliferative and pathogenic signaling cascades, including c-Myc, Fos, Jun, and cAMP ${ }^{21-23}$, are transactivated in cystic kidneys. Thus, the goal of this study was to define the epigenetic mechanisms that underlie this widespread aberrant gene expression. In this work, we perform a comparative H3K27ac ChIP-Seq analysis and provide a detailed enhancer/super-enhancer map of cystic kidneys. We find that mice and human ADPKD kidneys bear a similar enhancer signature. Moreover, we dissect and characterize a series of enhancers flanking the pathogenic c-Myc, Jun, and Fos genes. Finally, our studies suggest that enhancer rewiring is partly fueled by the metabolic reprogramming observed in ADPKD kidneys. Together, the epigenetic map unveiled by our studies has uncovered a series of potential new targets to slow cyst growth. 


\section{METHODS}

Mice: Ksp ${ }^{\mathrm{Cre}}, P k d 1^{\mathrm{F} / \mathrm{F}}, P k d 1^{\mathrm{RC} / \mathrm{RC}}, \mathrm{Pkhd1^{ \textrm {Cre } }}$ and $P k d 2^{\mathrm{F} / \mathrm{F}}$ mouse strains have been previously described ${ }^{24-28}$. The Ksp ${ }^{\mathrm{Cre}} ; P k d 1^{\mathrm{RC} / \mathrm{RC}}$ mice were bred with $P k d 1^{\mathrm{F} / \mathrm{F}}$ mice to obtain Cre-negative; $P k d 1^{\mathrm{F} / R C}$ (control) and Ksp $\mathrm{Cre} ; P k d 1^{\mathrm{F} / R C}$ (Pkd1-mutant) mice. Kidneys were harvested at the ages noted in results. All mice were maintained in C57BI/6 background. For ChIP experiments, each biological replicate consisted of four pooled kidneys from two mice of the same sex. Equal males and females were used in all studies. All experiments were performed according to the guidelines and approval of the UT Southwestern Institutional Animal Care and Use Committee.

Human Specimens: Human kidney specimens (ADPKD and NHK) were provided by the PKD Biomarkers and Biomaterials Core at the Kansas PKD center at the Kansas University Medical Center (KUMC). Kidneys were procured through the Bio-specimen Resource Core at the University of Kansas Cancer Center. Harvested kidneys were instantly sealed in sterile bags in the operating room, submerged in ice, and sent to the laboratory for processing. All surgical and processing protocols followed federal regulations and were approved by the Institutional Review Board at KUMC.

Cell culture: $P k d 1^{\mathrm{RC} /+}$ and $P k d 1^{\mathrm{RC} /-}$ cells were generated and maintained in previously described epithelial media with $2 \%$ fetal bovine serum (FBS) at $37^{\circ} \mathrm{C}^{29}$. Briefly, two kidneys were harvested from a 14-day old $P k d 1^{\mathrm{RC} / \mathrm{F}}$ male mouse and minced to $1 \mathrm{~mm}$ cubes. The tissue was incubated for 40 min in DMEM containing 5\% Collagenase (Sigma \#C1639, USA) at $37^{\circ} \mathrm{C}$ with intermittent agitation to create a single-cell suspension. Cells were strained using a 40-micron cell strainer and incubated with Biotinylated Dolichos Biflorus Agglutinin (DBA) (Vector labs \#B-1035) for 1 hour. DBA positive cells were isolated using a CELLection Biotin binder kit (Invitrogen \#11533D) and placed in culture media overnight. Next, cells were immortalized using the SV40 T Antigen Cell Immortalization Kit (Alstem \#CILV01) and underwent a clonal expansion in 96 well plates. Clones were screened for SV40 marker by genotyping for SV40, and one clone was selected for infection with an adenovirus that expresses Cre recombinase (Vector Biolabs \#1779). Infected 
cells subsequently underwent clonal expansion. Genotyping for the recombined Pkd1 allele confirmed $P k d 1^{\mathrm{RC} /-}$ genotype.

Chromatin Immunoprecipitation (ChIP): ChIP was performed using Simple ChIP Enzymatic Chromatin IP Kit (Cell Signaling \#9005). Briefly, tissues were minced and then cross-linked with $37 \%$ formaldehyde for 20 minutes. Specimens were then neutralized with glycine, washed with PBS and protease inhibitor cocktail, and then underwent lysis. Fragmentation was performed by micrococcal nuclease, and the lysate was obtained via mild sonication. Clarified lysate was incubated with $1 \mathrm{mg}$ of H3K27ac antibody (Abcam \#ab4729) or IgG overnight on a rotating platform kept at $4^{\circ} \mathrm{C}$. Before adding the antibody, $20 \mathrm{ul}$ of the lysate (2\%) was removed (input). To the enriched lysate, 30 ul of ChIP-grade Protein $\mathrm{G}$ was added and put on a rotating platform for $3 \mathrm{~h}$ at $4^{\circ} \mathrm{C}$. The chromatin-complex bound to beads was washed three times with low salt wash buffer and once with high salt wash buffer. The chromatin complex was then eluted from beads at $65^{\circ} \mathrm{C}$ with gentle shaking for 30 minutes. The protein-DNA cross-links were reversed, and the DNA was purified using DNA purification spin columns and recovered efficiently. The eluted DNA was quantified using Qubit ${ }^{\mathrm{TM}}$ dsDNA HS Assay Kit (Invitrogen \#Q32851, USA). The H3K27ac enriched regions were analyzed by quantitative real-time PCR and ChIP-seq. (Suppl. Table 2 and 3). KAPA HTP Library Preparation Kit was used to prepare libraries and samples were run on Illumina HiSeq 2500.

Analysis of ChIP-seq Data: The raw reads were aligned to the mouse reference genome (GRCh38/mm10) using default parameters in BWA v0.7.12 ${ }^{30}$. The aligned reads were subsequently filtered for quality, and uniquely mappable reads were retained for further analysis using Samtools version 1.6 and Sambamba version 0.6.6 ${ }^{31,32}$. Library complexity was measured using BEDTools version 2.26.0 and meets ENCODE data quality standards ${ }^{33,34}$. Relaxed peaks were called using MACS version 2.1 .0 with a $\mathrm{p}$-value of $1 \times 10^{-2}$ for each replicate, pooled replicates' reads, and pseudoreplicates ${ }^{35}$. Peak calls from the pooled replicates that were either observed in all replicates or both pseudoreplicates were used for subsequent analysis. 
Identification of variant enhancer loci: To identify enhancers, we first mapped transcription start sites (TSS) for protein-coding genes from Gencode version 15 annotations using MakeGencodeTSS (https://github.com/sdjebali/MakeGencodeTSS) ${ }^{36}$. Potential enhancers were defined as peaks that were $>2 \mathrm{~kb}$ away from known TSS, protein-coding genes. To detect differentially bound enhancers, we used the R package DiffBind version 2.2.12 with a p-value < 0.05 and default parameters.

Identification of super-enhancers loci: Super-enhancers were called using the ROSE version 0.1 package using the universe of $\mathrm{H} 3 \mathrm{~K} 27 \mathrm{ac}$ peaks called for each mouse model ${ }^{17,37}$. Differential super-enhancers were assessed using a universe of super-enhancers created by using the R package DiffBind version 2.2.12. Quantification of differential super-enhancers was assessed from using the master list and counting the overlapping differential enhancers previously identified using BEDTools version 2.26.033. We identified a differential super-enhancer if a given mouse model had at least one enhancer present. The gained and lost super-enhancers were identified to have more differential enhancers.

Generating Heatmaps and Metagenes: Enrichment of ChIP-seq signal was assessed by generating heatmaps and metagenes. For heatmaps and metagenes of ChIP-seq intensities, we used deepTools ${ }^{38}$. Read abundances were generated using 'computeMatrix' around the peak center and subsequently used to generate heatmaps and metagenes using 'plotHeatmap' and 'plotProfile', respectively.

Peak Annotations and Motif Enrichment: Peak annotations were identified by using the R 3.3.4 package ChIPseeker version1.1.18 using 'TxDb.Mmusculus.UCSC.mm10.knownGene' and 'org.Mm.eg.db' and default parameters ${ }^{39}$. To identify enriched motifs, we performed motif enrichment analysis by using HOMER version $4.9^{40}$. 
Analysis of Embryonic ChIP-seq data: Embryonic data sets with alignments and peak files for H3K27ac ChIP-seq experiments (ENCSR057SHA; E15.5) and (ENCSR178MLS; P0) were extracted from ENCODE. The datasets were analyzed following the same method as our H3K27ac ChIP-seq datasets.

SnATAC-seq Analysis: Publicly-available dataset GSE157079 was downloaded from Gene expression omnibus ${ }^{41}$. Minimum peak accessibility of each cell type was determined by comparing peak counts per cell type to a null peak count of the same total size using a one-tailed Fisher exact test. Those cell types with a $p$-value $<0.05$ for a peak were considered accessible at that peak. Differential peak accessibility of cell types was scored by comparing the peak counts per cell type to those of all other cell types using a two-tailed Fisher exact test with BenjaminiHochberg p-value correction. If a cell-type pair comparison for a peak has an adjusted p-value $<0.05$, then the upregulated cell type's score was incremented.

TAD-data association: RNA-seq and ChIP-seq datasets were binned into the topologically associated domains (TAD) of mouse embryonic stem cells (mESC) $)^{42}$. The midpoint of each transcript or peak was used to determine the TAD assignment.

RNA extraction and qPCR: RNA extraction was performed with miRNeasy Kit (Qiagen \#217004). Superscript III (Invitrogen) was used for the synthesis of first-strand cDNA from mRNA. qPCR was performed using the iQ SYBR Green Supermix (Bio-Rad) using the CFX ConnectTM Real-time PCR detection system. All samples were analyzed with technical replicates. Ribosomal $18 \mathrm{~S}$ was used to normalize mRNA expression.

Generation of CRISPR-KO cell lines: SgRNAs were designed to flank enhancers and cloned

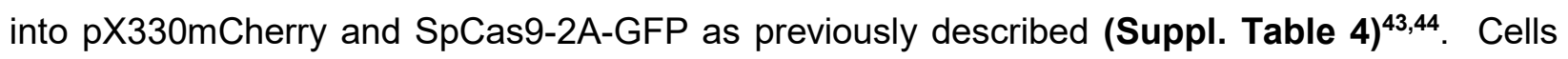
were transfected using Lipofectamine 3000 Reagent (Invitrogen) and underwent FACS sorting 72 hours after transfection to select for double-positive cells and subsequent single-cell plating in 96 
well plates. Clonal cell lines were genotyped (Suppl Table 5) to assess for deletion of each respective enhancer. In each experiment, clonal cell lines which underwent transfection with sgRNAs but did not develop deletion of enhancers were utilized as control cell lines for subsequent experiments.

Western blot: Total protein was extracted with Tissue Protein extraction reagent (T-PER, Thermofisher Scientific, USA) + Protease inhibitor cocktail (Pierce \#A32961). Protein concentration was measured using Bradford's reagent. Ten to twenty micrograms of denatured protein was loaded onto a 4-20\% TGX stain-free polyacrylamide gel (Bio-Rad, USA) or NuPAGE 3-8\% Tris acetate gel and subsequently transferred onto pre-cut nitrocellulose membranes. The membrane was blocked in $5 \%$ milk for $1 \mathrm{~h}$ followed by incubation with primary antibodies: c-Myc (Abcam ab185656; 1:1000), Fos (Cell Signaling 2250S; 1:1000), Jun (Cell Signaling 9165; 1:1000), CREB (Cell Signaling 9197S; 1:1000), STAT1 (Cell Signaling 14994S; 1:1000), PC1(7e12) (Santa Cruz \#SC-130554 1:1000), PC2 (Baltimore PKD Center) or Actin (Sigma a3854;1:40,000) overnight or $30 \mathrm{~min}$ at $4^{\circ} \mathrm{C}$. The blots were washed with TBST and probed with Goat anti-Rabbit-HRP conjugated secondary antibody for $1 \mathrm{~h}$. The blots were washed three times with TBST prior to development with SuperSignal West Femto, SuperSignal West Dura Extended Dilution Substrate, or ECL.

Alamar Blue assay: $1 \times 10^{4}$ cells were plated in 24 -well plates. The following day, fresh media containing $10 \%$ Alamar Blue reagent was added. Absorbance at 570 and $600 \mathrm{~nm}$ was measured using BioTek Synergy H1 microplate reader 12 hours post-incubation, and percentage reduction was determined.

3D Cystogenesis Assay: Matrigel basement membrane matrix (Corning, \#354234) was thawed on ice for $30 \mathrm{~min}$. Micropipette tips and 8-well chamber slides were pre-cooled at 4 degrees. 8well LAB-TEK II chamber slides w/ cover glass (Corning \# 155409) were placed on ice and coated 
with $25 \mu \mathrm{l} 100 \%$ matrigel and then placed at 37 degrees. Cells were trypsinized, counted, and diluted to $1.5 \times 10^{4}$ cells $/ \mathrm{ml}$ in epithelial media. Matrigel was diluted in epithelial media to achieve a final concentration of $4 \%$. The cell suspension and $4 \%$ matrigel were mixed (1:1) to achieve a final concentration of $2 \%$ matrigel. $300 \mu$ of cell/matrigel mix was added to each well. 7 days postseeding, 20x images of cysts were obtained using light microscopy and quantified with Image $\mathrm{J}$ software. For each experiment, 100 cysts were quantified. Each experiment was repeated 3 times (biological replicates).

Histone extraction: Histones were extracted via an acid-extraction method. Briefly, samples were weighed, cut, and minced with a blade. The minced tissue was then transferred to a Dounce homogenizer and re-suspended in TEB buffer (PBS with 0.5\% Triton X 100, $2 \mathrm{mM} \mathrm{PMSF,} \mathrm{and}$ $0.02 \% \mathrm{NaN}_{3}$ ) at $0.5 \mathrm{ml}$ per $100 \mathrm{mg}$ tissue and disaggregated by 50 strokes. The homogenate was transferred to a $2 \mathrm{ml}$ Eppendorf tube and centrifuged at $10,000 \mathrm{rpm}$ for $1 \mathrm{~min}$ at $4^{\circ} \mathrm{C}$. The cells were harvested and centrifuged at $1000 \mathrm{rpm}$ for 5 minutes at $4^{\circ} \mathrm{C}$. The cell pellet was resuspended in TEB buffer at $10^{7}$ cells $/ 0.5 \mathrm{ml}$ and lysed on ice for 10 minutes via vortex. The cell lysates were centrifuged at $10,000 \mathrm{rpm}$ for 1 minute at $4^{\circ} \mathrm{C}$. The supernatant was removed, and the cell/tissue pellet was re-suspended in 3 volumes (approx. $100 \mu \mathrm{l} / 10^{7}$ cells or $100 \mathrm{mg}$ tissues) of extraction buffer $(0.5 \mathrm{~N} \mathrm{HCl}+10 \%$ glycerol $)$ and was incubated on ice for $30 \mathrm{~min}$. The cell lysate was centrifuged at $12,000 \mathrm{rpm}$ for 5 minutes at $4^{\circ} \mathrm{C}$, and the supernatant was transferred to a new vial. To the supernatant, approximately eight volumes $\left(0.3 \mathrm{ml} / 10^{7}\right.$ cells or $100 \mathrm{mg}$ tissues $)$ of acetone was added and stored at $-20^{\circ} \mathrm{C}$ overnight. The supernatant-acetone mixture was centrifuged at 12,000 rpm for 5 minutes and air-dried for 10-20 min. The pellet was dissolved in distilled water (30 $\mu \mathrm{l} / 10^{7}$ cells or $100 \mathrm{mg}$ tissues). Bradford assay was used to quantify histone abundance for subsequent use in ELISA assay.

Histone H3 acetyl Lys27 ELISA: The Histone H3 acetyl Lys27 ELISA (H3K27ac) kit (Active Motif, \#53116) was used to detect H3K27ac levels from crude acid extracted histone preparations. 
Briefly, the sandwiched ELISA kit uses a 96-well strip coated with H3K27ac monoclonal antibody for capturing histone $(\mathrm{H} 3)$ from samples followed by incubation with anti-mouse H3K27ac primary antibody (1:500). A secondary antibody conjugated with HRP (1:500) and developing substrate is used for colorimetric detection at $450 \mathrm{~nm}$. A recombinant H3K27ac protein provided in the kit was used to plot a reference standard curve to quantify the amount of H3K27ac present in each sample.

Immunostaining: Paraffin-embedded sections were used for mouse kidney tissues. In the case of human kidney tissues, frozen sections were used. Both anti-H3K27ac (Abcam \#ab4729) and Alexa Fluor anti-Rabbit 594 secondary antibodies were used at 1:500 dilution for immunostaining experiments.

Metabolism Experiments: $P k d 1^{\mathrm{RC} /-}$ cells were grown to $60 \%$ confluency and then incubated with $20 \mu \mathrm{M}$ 2-DG for 24 hours, $40 \mu \mathrm{M}$ BPTES for 48 hours, or $40 \mu \mathrm{M}$ of $\mathrm{WY}-14643$ for 72 hours. Corresponding vehicle controls also underwent equivalent incubation times before harvest for ChIP, ELISA, and Western blot analysis.

Antisense Oligonucleotide Treatment: Antisense oligonucleotides (ASOs) were designed and obtained from Qiagen. $2 \times 10^{\wedge 5}$ cells were seeded in a 6 well plate, and the following day media was changed. Lipofectamine 3000 was used to transfect $40 \mathrm{nM}$ scramble ASO or Junos ASO (cocktail of 3 ASOs targeting 3 different regions of the Junos transcript at final total concentration of $40 \mathrm{nM}$ ). Fluorescent microscopy was performed after 24 hours to confirm adequate delivery of ASO by visualization of FAM. Cells were harvested after 72 hours for subsequent molecular analysis.

Data Availability: The ChIP-seq data are available at Geo Expression Omnibus GSE189153. 
bioRxiv preprint doi: https://doi.org/10.1101/2021.11.19.469306; this version posted November 19, 2021. The copyright holder for this preprint (which was not certified by peer review) is the author/funder. All rights reserved. No reuse allowed without permission.

Statistical Analysis: For statistical analysis, a two-tailed Student's t-test for pairwise comparison was performed. For 3D cyst assay and Alamar blue experiments, a nested t-test was performed. $P<0.05$ was considered statistically significant. 


\section{RESULTS}

H3K27ac histone modification is increased in mouse and human ADPKD samples: Active enhancers are marked by H3K27ac, an acetylation of lysine residue (27 $7^{\text {th }}$ position) of the DNA packing protein Histone $\mathrm{H}^{45}$. Therefore, to assess the epigenetic status of cystic kidneys, we began by measuring global H3K27ac abundance in kidneys of orthologous ADPKD mouse models. We extracted histones from whole kidneys of 18-day-old Ksp ${ }^{\mathrm{Cre}} ; P k d 1^{\mathrm{F} / \mathrm{RC}}$ (Pkd1-mutant), 28-day-old Pkhd11re; $P k d 2^{\mathrm{F} / \mathrm{F}}(P k d 2-\mathrm{KO})$, and their respective age-matched littermate control mice. We then used ELISA to quantify H3K27ac levels within the extracted histones. We found that $\mathrm{H} 3 \mathrm{~K} 27$ ac levels were $55 \%$ higher in Pkd1-mutant and $17 \%$ higher in $P k d 2-\mathrm{KO}$ kidneys compared to their respective age-matched control kidneys (Fig. 1A-B). Moreover, staining with an anti-H3K27ac antibody also revealed a higher H3K27ac immunofluorescence signal. Quantification of random 20x images demonstrated that the H3K27ac signal was higher by 55\% in Pkd1-mutant kidneys (Fig. 1D-E and L) and by 64\% in Pkd2-KO kidneys compared to their respective control kidneys (Fig. 1F-G and $\mathbf{M}$ ). We also measured H3K27ac abundance in the long-lived, slowly progressive Pkd1 RC/RC ADPKD mouse model. Compared to age-matched controls, the H3K27ac level was increased in 160 -day-old $P k d 1^{\mathrm{RC} / R C}$ mice kidneys by 55 percent (Fig. 1H, I, and N). Notably, the predominant increase in the H3K27ac signal in each mouse model originates from the cyst epithelial cells.

To determine whether our observations in mouse models are relevant to human ADPKD, we measured H3K27ac levels using ELISA $(\mathrm{N}=5)$ and immunofluorescence $(\mathrm{N}=3)$ in normal human kidney (NHK) samples and cystic kidney samples from individuals with ADPKD. Similar to murine ADPKD models, ELISA-measured H3K27ac level was increased in human ADPKD samples by $64 \%$ compared to NHK controls (Fig. 1C). Moreover, immunofluorescence staining of kidney sections from a different group of NHK and ADPKD kidney samples 
demonstrated higher H3K27ac signal primarily enriched in cyst epithelial cells (Fig. 1J and K and Suppl. Fig. 1). Thus, global H3K27ac levels are increased in both mouse and human ADPKD.

Comparative H3K27ac ChIP-Seq uncovers the enhancer landscape of cystic kidneys: Our observation of higher H3K27ac levels in ADPKD models points to substantial epigenetic rewiring in cystic kidneys. As the first step towards identifying the enhancer landscape of cystic kidneys, we immunoprecipitated and sequenced DNA (ChIP-Seq) bound to H3K27ac-modified histones in 16-day-old control and Pkd1-mutant kidneys ( $\mathrm{N}=3$, each replicate consisted of chromatin pooled from four kidneys) (Fig. 2A). We used 16-day-old Pkd1-mutant kidneys because they are mildly cystic, allowing us to identify enhancers in the early stages of cystogenesis. Principal component analysis revealed that control and Pkd1-mutant ChIP-Seq samples clustered separately (Suppl Fig. 2A). We cross-compared the H3K27ac-modified epigenome of control and cystic kidneys. In concordance with our findings of higher total H3K27ac levels, we found that 16560 regulatory elements were gained (increased H3K27ac), and only 1552 were lost (decreased H3K27ac) in Pkd1-mutant compared to control kidneys (Fig. 2B). Moreover, annotation of these regulatory elements revealed that $93 \%$ of the H3K27ac ChIP-seq peaks were located in intron or intergenic regions (Fig. 2C). Only $2.8 \%$ of peaks were found in promoter regions indicating that the gain in the H3K27ac signal was primarily derived from the development of new enhancer elements. We next validated our ChIP-Seq results by performing ChIP-qPCR analysis of arbitrarily selected gained and lost regulatory elements in a new cohort of 16-day-old control and Pkd1 mutant kidneys (Suppl Fig. 2B). Furthermore, to determine if our ChIP-Seq results can be extrapolated to other orthologous ADPKD models, we also performed H3K27ac ChIP using 21-day-old control and Pkd2-KO kidneys. We were able to validate Pkd1-mutant ChIP-Seq data even in Pkd2-KO kidneys, suggesting similar epigenetic rewiring in both ADPKD models (Suppl Fig. 2C).

The transcriptomic profile of cystic kidneys has been likened to that of a developing kidney ${ }^{46}$. Therefore, we examined whether cystic and developing kidneys also share a similar epigenetic 
profile. We used ENCODE consortium H3K27ac ChIP-Seq datasets and mapped enhancers in embryonic $(E) 15.5$ and postnatal day $(P) 0$ kidneys ${ }^{47,48}$. We then cross compared the ENCODE and our datasets and found that P16 non-cystic control kidneys have little resemblance to developing E15.5 or P0 kidneys. In contrast, we noted that Pkd1-mutant kidneys share $42 \%$ and 32\% of H3K27ac peaks with E15.5 and P0 kidneys, respectively (Fig. 2D and Suppl Fig. 3A-B). Next, we deconvoluted our bulk H3K27ac ChIP-Seq data by integrating a recently published single-cell ATAC-seq dataset of wildtype mouse E18.5 kidney $^{41}$. ATAC-seq is utilized to catalog genome-wide open chromatic positions, which is another indicator of active regulatory elements. In concordance with our findings of similarities with developing kidneys, $64 \%$ of active enhancers in 16-day-old Pkd1-mutant kidneys were found in regions with appropriately open/closed chromatin in the E18.5 kidneys (Suppl Fig. 3C). Moreover, this analysis revealed that our bulk H3K27ac signal primarily mapped to open chromatin locations in tubular epithelial and stromal cells (Fig. 2E).

Enhancers regulate gene expression by interacting with nearby or very distant promoters. However, topological associated domain (TAD) boundaries serve as physical limits on the range of enhancer-promoter interaction ${ }^{42}$. To determine the influence of enhancers on dysregulated gene expression in PKD, we first overlapped the H3K27ac ChIP-Seq data with our previously published RNA-seq dataset from 22-day-old control and Pkd1-mutant kidneys ${ }^{21}$. Next, we examined the relationship between active enhancers and dysregulated genes located within the same TAD boundaries. We found that $90 \%$ of upregulated genes in Pkd1-mutant kidneys were located in a TAD that also housed a gained enhancer (Fig. 2F, G). Conversely, 64\% of downregulated genes were found in a TAD that had lost an enhancer. Finally, pathway analysis of the positively-correlated enhancer-dysregulated gene pairs revealed cancer signaling as the top influenced network (Fig. 2H). Closer examination of genes within the cancer signaling 
pathway identified $c-M y c$ as the top gene with the most associated enhancers. Taken together, our analysis has uncovered a large repertoire of regulatory enhancers in cystic kidneys.

\section{Evolutionarily-conserved enhancer cluster transactivates c-Myc expression in cellular}

ADPKD model: The proto-oncogene $c-M y c$ is transactivated in mouse and human ADPKD, and its overexpression is sufficient to induce kidney cysts in mice $21,49,50$. However, how c-Myc expression is regulated in PKD is incompletely understood. We identified 30 regulatory elements spanning 2.5 Mbp genomic region surrounding the $c-M y c$ gene that displayed significantly enriched H3K27ac signal in Pkd1-mutant kidneys compared to control kidneys (Fig. 3A). A query of the publicly available high-throughput chromatin conformation capture $(\mathrm{Hi}-\mathrm{C})$ datasets revealed that these 30 active enhancers physically interact with the c-Myc promotor (Fig. $3 \mathbf{A})^{51}$. We found that $22 / 30$ enhancers are evolutionarily-conserved between mice and humans. Ten of these enhancers have not previously been reported in the literature (Supplementary Table 1$)^{52}$. These observations suggest that the gained enhancers may underlie c-Myc upregulation in ADPKD and prompted us to further validate this locus. We began by performing ChIP-qPCR in an independent cohort (biological replicates) of 16-day-old control and Pkd1-mutant kidneys and confirmed that 20 conserved enhancers indeed display higher H3K27ac levels in cystic kidneys (Fig. 3B). As a pertinent negative control, we found several regions that exhibit equal H3K27ac signal in our ChIP-Seq data remained unchanged by ChIP-qPCR validation in control and Pkd1-mutant kidneys (Fig. 3B). Next, we asked which of these enhancers were also enriched in Pkd2-KO kidneys. ChIP-qPCR revealed that 13/20 enhancers have higher H3K27ac signal in 21-day-old Pkd2-KO kidneys compared to age-matched control kidneys (Fig. 3C). Finally, we examined whether these enhancers are relevant to human ADPKD. ChIP-qPCR showed that 12/20 enhancers exhibited higher H3K27ac modification in the human ADPKD samples than NHK control samples (Fig. 3D). Taken together, our careful assessment of the $c-M y c$ locus identified 
12 evolutionarily-conserved enhancers that are activated in both mouse and human ADPKD samples.

To determine if these enhancers drive c-Myc expression, we used CRISPR/Cas9 to delete them in a cellular $P k d 1^{\mathrm{RC} /-}$ ADPKD model. The $P k d 1^{\mathrm{RC} /-}$ cells are of collecting duct origin (see methods for details) that harbor a hypomorphic p.R3277C mutation on one Pkd1 allele, whereas the other is deleted ${ }^{53}$. Isogenic $P k d 1^{\mathrm{RC} /+}$ cells serve as controls. Our characterization of this new cellular model revealed that it recapitulates the key pathogenic hallmarks of Pkd1-mutant mice. We found that $P k d 1^{R C /}$ cells have reduced Polycystin-1 (but not Polycystin-2) expression (Fig. 4A) and higher 3D cyst growth (Fig. 4B), and proliferation rates (Fig. 4C) than $P k d 1^{R C /+}$ cells. Importantly, we noted activated pathogenic ADPKD signaling, including higher c-Myc, Fos, and CREB expression in $P k d 1^{R C /-}$ compared to $P k d 1^{R C /+}$ cells (Fig. 4A and Suppl. Fig 4). Moreover, as is the case with mouse and human ADPKD samples, ELISA revealed $35 \%$ higher total H3K27ac levels in $P k d 1^{R C /-}$ cells compared to $P k d 1^{R C /+}$ cells (Fig. 4D). Moreover, ChIP-qPCR showed increased H3K27ac modification on 10/22 conserved c-Myc-flanking enhancers in $P k d 1^{\mathrm{RC} /-}$ cells compared to $P k d 1^{\mathrm{RC} /+}$ cells (Fig. 4E).

The enhancer elements are referred to as E1 through E30, labeled based on their genomic position (in 5'-3' orientation) within the TAD, which houses the $c-M y c$ gene. First, we deleted the $3 \mathrm{~kb}$ region, encompassing the $\mathrm{E} 19$ enhancer in $P k d 1^{R C /-}$ cells. We generated four independent clonal cell lines lacking the E19 enhancer, and in all four cell lines, we noted a reduction in c-Myc expression (Fig. 4F, Suppl. Fig. 5A). We also noted that 3D cyst growth was reduced by $50 \%$ in E19 null clones compared to their respective unedited $P k d 1^{\mathrm{RC} /-}$ parental clones (Fig. 4G). Moreover, cellular proliferation as measured by the Alamar Blue assay was reduced, and western blot analysis revealed lower PCNA levels in each of the E19 null clones compared to unedited clones (Fig. 4H). Next, we generated two clonal cell lines that lacked a $1.6 \mathrm{~kb}$ region encompassing E27 and E28 enhancers (Suppl Fig 5B). Again, we noted reduced c-Myc and 
PCNA expression (Fig. 4I), a 30\% reduction in cyst size (Fig. 4J), and a lower proliferation rate (Fig. 4K) in E27-28 deleted $P k d 1^{\mathrm{RC} /-}$ cell lines compared to their unedited counterparts. We also deleted the genomic locus containing E5-E9 enhancers (Suppl Fig. 6A). However, this deletion was not sufficient to reduce Myc. Finally, we attempted to delete three additional loci: $4.9 \mathrm{~kb}$ genomic region containing E21-22, 868 bp genomic region containing E25, and 498bp genomic region containing E29. However, we could only recover heterozygous deletions of E21-22 and E25 (Suppl Figure 6B,C). We did not observe differences in c-Myc expression in any of the heterozygous deletion lines. Similarly, we did not obtain even heterozygous deletion of the genomic region encompassing E29 despite multiple attempts. Thus, genomic loci bearing E19, E27, and E28 enhancers promote c-Myc expression in cellular ADPKD models.

Super-enhancers promote Fos and Jun expression: Super-enhancers are large genomic regions that contain multiple enhancers in close proximity to each other. We used the ROSE (Rank Ordering of Super-Enhancers) bioinformatic tool to map ADPKD-relevant super-enhancers from our H3K27ac ChIP-Seq dataset ${ }^{17,37}$. Using differential binding analysis, we found that Pkd1mutant kidneys gained 101 (higher H3K27ac) and lost 5 (lower H3K27ac) super-enhancers compared to control kidneys (Fig. 5A-B). These super-enhancers, on average, were $71 \mathrm{~kb}$ in genomic length and exhibited greater than 50-fold higher H3K27ac modification compared to a typical enhancer (Fig 5C and D). We also mapped the super-enhancer landscape of E14.5, E16.5, and P0 developing kidneys using the ENCODE data. As with enhancers, we found that many Pkd1-mutant gained super-enhancers were also active in developing kidneys (Fig. 5E). Interestingly, cross-comparison with the dbSUPER super-enhancer database revealed that numerous gained super-enhancers were enriched in stromal and immune cells, suggesting epigenetic rewiring even in the cyst microenvironment (Fig. 5F). Finally, motif analysis revealed that the AP-1 binding element was significantly enriched amongst the gained super-enhancers (Fig 5G). 
AP-1 is a tetramer transcription factor comprised of Jun and Fos. Interestingly, we noted that Jun and Fos loci themselves are flanked by gained super-enhancers. Considering both Jun and Fos are implicated in mouse and human ADPKD, we asked whether these super-enhancers are relevant to cyst growth. First, ChIP-qPCR in control and Pkd1-mutant kidneys confirmed higher H3K27ac modification on Fos, Jun, and a series of other randomly selected superenhancers (Fig. 5H). Moreover, we found this super-enhancer landscape was also observed in Pkd2-mutant kidneys, $P k d 1^{\mathrm{RC} /-}$ cells, and human ADPKD samples compared to their respective controls (Fig. 5l-K). The Fos super-enhancer encompasses a $35 \mathrm{~kb}$ region upstream of the Fos gene promoter (Fig. 6A). We used CRISPR/Cas9 to delete the upstream super-enhancer while leaving the Fos promoter intact in $P k d 1^{\mathrm{RC} /-}$ cells. Compared to unedited $P k d 1^{\mathrm{RC} /-}$ cells, we found that $P k d 1^{\mathrm{RC} /-}$ cells with a heterozygous deletion of the Fos super-enhancer had reduced Fos expression (Fig. 6B). Moreover, the edited $P k d 1^{R C /-}$ cells had lower cellular proliferation and cyst growth rates compared to the unedited $P k d 1^{\mathrm{RC} /-}$ cells (Fig. 6C and D).

The putative Jun super-enhancer is an uncharacterized $62 \mathrm{~kb}$ genomic region upstream of the Jun gene. Closer examination revealed that this super-enhancer region also encodes a long noncoding RNA (IncRNA) annotated as Junos (Fig. 6E). Therefore, the locus may act as a Jun super-enhancer, or alternatively, it could regulate Jun expression via Junos. To determine whether the IncRNA regulates Jun expression, we inhibited Junos in Pkd1 ${ }^{\mathrm{RCl} / \text { cells }}$ using antisense oligos (ASOs) while still keeping the super-enhancer intact (Fig. 6F). ASO treatment reduced Junos level by 68 percent (Fig. 6G). However, Jun expression remained unchanged, indicating that the IncRNA does not regulate Jun expression (Fig. 6H-I). Next, we used CRISPR/Cas9 and generated three clonal cell lines with homozygous deletion of the $70 \mathrm{~kb}$ super-enhancer region. Compared to the unedited $P k d 1^{R C /-}$ control cells, Jun expression was reduced in all three $P k d 1^{R C /-}$ cell lines lacking the super-enhancer (Fig. 6J). Moreover, cellular proliferation and cyst size were also significantly reduced in cell lines Jun super-enhancer deletion (Fig. 6K-L). Thus, both 
components of the AP-1 complex are trans-activated by neighboring super-enhancers in Pkd1mutant cells.

ADPKD metabolic pathways influence H3K27ac levels. Histone acetylation is a dynamic process tied to acetyl-CoA availability, which is controlled by the cellular metabolic state ${ }^{54-56}$. ADPKD is marked by extensive metabolic reprogramming, including the activation of aerobic glycolysis and glutaminolysis and inhibition of oxidative phosphorylation. Therefore, we asked whether the ADPKD metabolic pathways regulate H3K27ac levels. First, we treated Pkd1 ${ }^{\mathrm{RC} / \text { - cells }}$ with 2-deoxyglucose (2-DG) for 24 hours and then measured H3K27ac by ELISA. 2-DG is a competitive inhibitor of aerobic glycolysis. We noted that 2-DG treatment in $P k d 1^{\text {RCl- }}$ cells reduced H3K27ac levels by $30 \%$ (Fig. 7A). Moreover, H3K27ac modification was reduced at c-Myc enhancers with 2-DG treatment in Pkd1 $1^{\mathrm{RC} /-}$ cells (Fig. 7B). Accordingly, c-Myc expression was

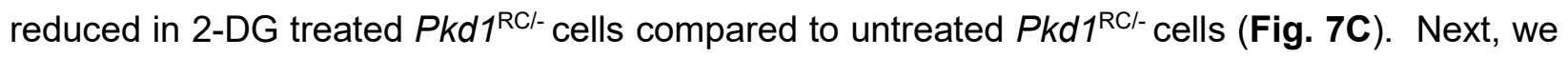
treated $P k d 1^{\mathrm{RC} /-}$ cells with BPTES, an allosteric inhibitor of the glutaminase enzyme, to turn off glutaminolysis or WY-14643, a Ppara agonist, to activate oxidative phosphorylation. We noted that compared to untreated Pkd1 ${ }^{\mathrm{RC} /-}$ cells, total H3K27ac levels and H3K27ac abundance at c-

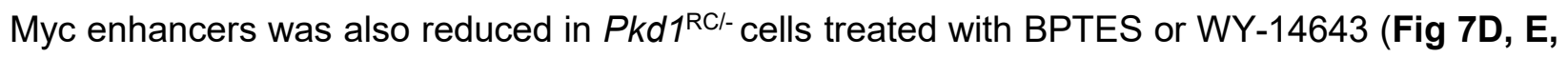
G, and H). Accordingly, Western blot analysis revealed that treatment with both BPTES and WY14643 reduced $c-$ Myc expression (Fig 6F and I). Thus, the rewiring of the epigenetic profile is at least in part mediated by ADPKD metabolic reprogramming. 


\section{DISCUSSION}

Large-scale transcriptomic rewiring is a prominent pathological feature of ADPKD. Here, we provide an in-depth view of the cystic kidney enhancer landscape that underlies this dysregulated gene expression. The first novel insight of our work is that there is a marked increase in global H3K27ac levels in mouse and human ADPKD tissues. Further, our findings suggest this increase, at least in part, is a consequence of ADPKD metabolic reprogramming involving aerobic glycolysis and glutaminolysis. Indeed, both metabolic pathways are known to replenish and exaggerate the cellular acetyl-CoA pool, which in turn is the critical determinant for histone acetylation ${ }^{54-56}$. Conversely, work from others suggests that super-enhancers propagate the aberrant expression of metabolic pathway genes in Pkd1-mutant cells ${ }^{57}$. Thus, there may be a vicious metabolismepigenetics cycle in ADPKD, where the altered metabolism reshapes the epigenetic state of cyst epithelia, leading to a transcriptomic output conducive to sustained metabolic reprogramming.

The second and perhaps the most important outcome of our studies is identifying the genome-wide enhancer profile of Pkd1-mutant kidneys. We uncovered $>16,000$ preferentially activated CRE, including 105 super-enhancers, providing the first glimpse of extensive epigenetic rewiring of cystic kidneys. Some noteworthy observations were that: (i) the activated CREs are located in intergenic as well as intronic regions, implying both long and short-range enhancerpromoter looping events in PKD. (ii) Deconvolution of our global H3K27ac ChIP-seq data using E18.5 wildtype kidney scATAC-seq data suggested that activated enhancers are observed both in cyst epithelia and cyst microenvironment component stromal and immune cells. (iii) We noted that $>90 \%$ of upregulated genes are located in TADs that also housed activated enhancers suggesting that majority of the dysregulated transcriptome is facilitated by the altered epigenetic landscape. 
c-Myc and the AP-1 heterodimer complex components Fos and Jun are transactivated in ADPKD models ${ }^{22}$. In turn, these transcription factors activate pro-proliferative gene networks that are thought to underlie cyst growth ${ }^{58,59}$. Our work provides several new mechanistic insights into the regulation of this transcriptional circuitry. Our data suggest that a series of intergenic enhancers are necessary to drive c-Myc upregulation in ADPKD. Consistent with these observations, enhancer-mediated upregulation of c-Myc has also been reported in malignancies $^{52}$, albeit the enhancer choice appears to vary. Interestingly, 10/12 activated enhancers in both mouse and human ADPKD samples have not been reported in cancers, suggesting that c-Myc enhancer repertoire and choice may differ based on the type of tissue and disease. In support of this notion, deletion of a large enhancer 1.7 megabases downstream of the c-Myc gene abolishes c-Myc expression but only in hematopoietic stem cells and appears to be critical for leukemic transformation ${ }^{60}$. Therefore, it is tempting to speculate that the 10 ADPKDenriched enhancers double as regulators of c-Myc expression during kidney development. Moreover, these key enhancers could serve as novel targets to reduce c-Myc expression preferentially in the kidney and slow cyst growth.

Thematically similar to the c-Myc locus, we found that super-enhancer elements are necessary to upregulate Jun and Fos in Pkd1-mutant cells. The same Fos super-enhancer is also functional in neuronal cells suggesting that this CRE is active in diverse cell types ${ }^{61}$. In contrast, little was known about the $62 \mathrm{~kb}$ super-enhancer downstream of Jun. We report that this locus serves dual functions of a Jun super-enhancer and Junos IncRNA gene. Super-enhancerassociated IncRNAs are implicated in cis-activating nearby genes via mechanisms such as assisting in chromatin looping or transcription factor binding. Instead, we found that Junos was dispensable for Jun expression, implying an alternative biological role of this IncRNA, perhaps in trans-activating other target genes. 
There are limitations and caveats related to our work. First, TAD-specific active enhancer and upregulated gene pairs indicate positive correlation but do not imply a direct causal link. While we established causation for c-Myc, Fos, and Jun, all other enhancer-gene pairs remain unvalidated. High throughput CRISPR-based and massively parallel reporter assays have been recently described to study regulatory functions of enhancers ${ }^{62}$. Our dataset will be a valuable resource to apply these approaches. Second, integrating our H3K27ac ChIP-seq dataset with the Hi-C dataset provides a good view of the extensive chromatin looping in Pkd1-mutant kidneys. However, an independent $\mathrm{Hi}-\mathrm{C}$ dataset for cystic kidneys is needed for a refined and accurate description of the 3D chromatin architecture in PKD. Third, we did not validate c-Myc, Jun, or Fos CREs in ADPKD mouse models. These studies will require new mutant mouse models and are the focus of our future work. Finally, we observed congruent epigenetic signatures across the various ADPKD models and human samples based on ChIP-qPCR of arbitrarily selected enhancers. However, independent genome-wide datasets need to be developed in other ADPKD models, including human ADPKD tissues.

In summary, we provide the first insights into the enhancer map of Pkd1-mutant kidneys. We identify a series of enhancers near pro-proliferative transcription factors and demonstrate their ability to regulate cyst growth and gene expression. Our work also suggests that H3K27ac levels are regulated by rewired metabolism of Pkd1-mutant cells. Finally, developing pharmacological approaches to inhibit these enhancers could serve as a novel method to slow cyst growth. 


\section{Disclosures: None}

\section{Acknowledgments:}

This work is dedicated to the memory of Ms. Peyton Johnson, who we lost in 2020. We are grateful for her technical and intellectual contributions in this project. We thank Chun-Mien Chang for help with 3D cystogenesis, the PKD Research and Biomaterials and Cellular Models Core at the University of Kansas Medical Center for human tissue samples, and the McDermott Center Next Generation Sequencing Core and Bioinformatics Core Facility (BICF) at UT Southwestern Medical Center for providing critical services.

Work from the authors' laboratory is supported by National Institute of Diabetes and Digestive and Kidney Diseases Grants K08DK117049 (to R.L) and R01 DK102572 (to V.P.). R. L. is also supported by grants from the PKD Foundation and American Society of Nephrology KidneyCure Grants Program. V.M. is supported by the Cancer Prevention and Research Institute of Texas (RP150596) 


\section{FIGURES}
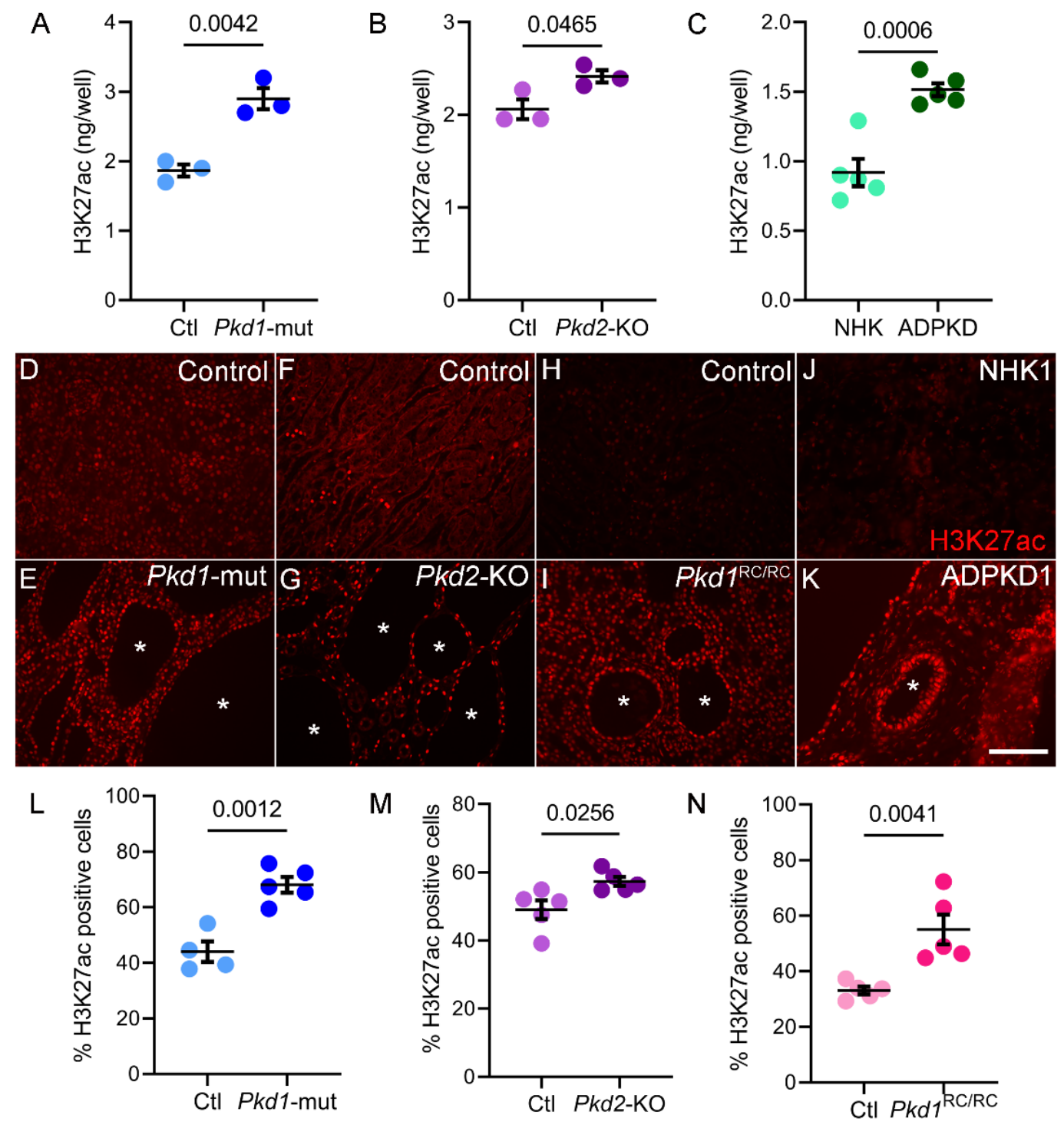

Figure 1. Global H3K27ac level is increased in ADPKD models. A and B. ELISA showing higher H3K27ac levels in 18-day-old Pkd1-mutant and 21-day-old Pkd2-KO kidneys compared to their respective age-matched control kidneys $(\mathrm{N}=3)$. C. ELISA showing higher H3K27ac levels in human ADPKD samples compared to normal human kidney (NHK) samples ( $N=5)$. D-K Representative images of immunofluorescence staining with anti-H3K27ac antibody in kidney sections of 18-day-old Pkd1-mutant, 21-day-old Pkd2-KO, 160-day-old $P k d 1^{R C / R C}$, and human ADPKD samples compared their respective controls. L-N. Quantification of the H3K27ac signal using the Image $\mathrm{J}$ software is shown. Error bars indicate SEM; * cyst; scale bar $=100 \mu \mathrm{M}$; Statistical analysis: Student's $t$-test. 
A
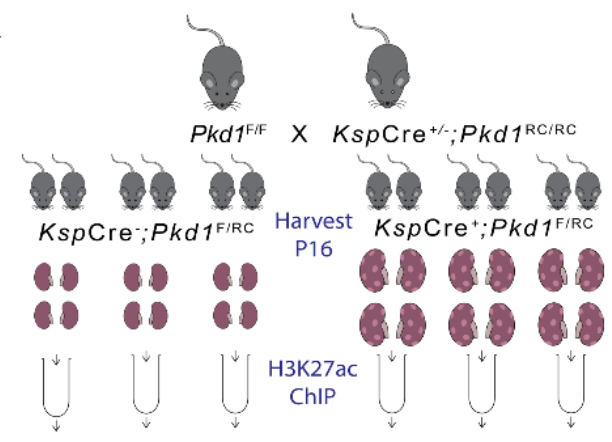

Library Preparation / Next-Generation Sequencing

D

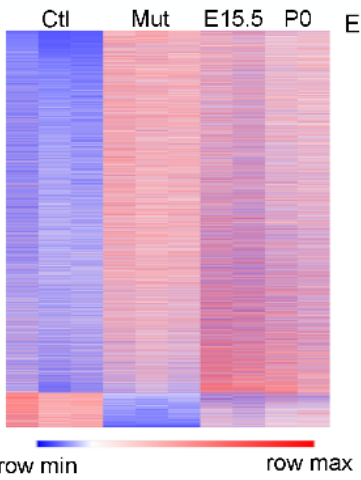

B

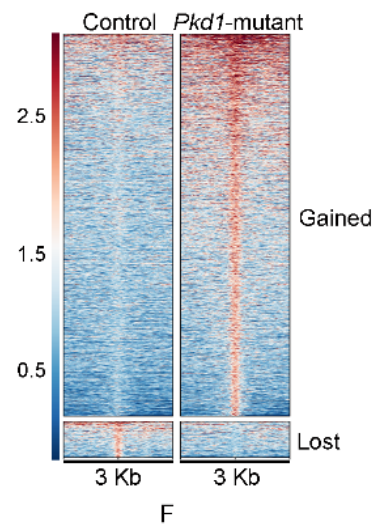

C

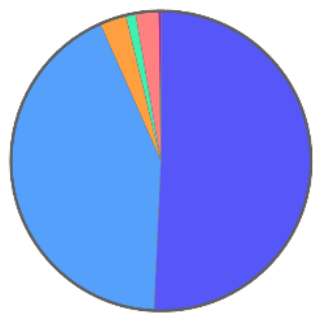

- Intron $(\mathrm{N}=9190)$

$\square$ Intergenic $(\mathrm{N}=7721)$

$\square$ Promoter $(\mathrm{N}=514)$

$\square$ Exon $(\mathrm{N}=192)$

$\square$ 3'-UTR ( $N=454)$

5'-UTR $(\mathrm{N}=43)$
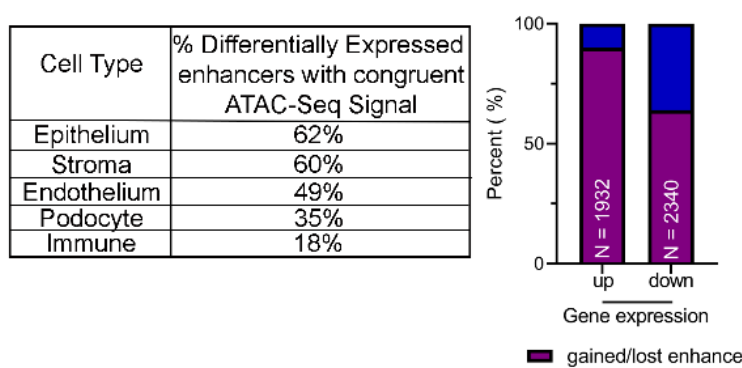
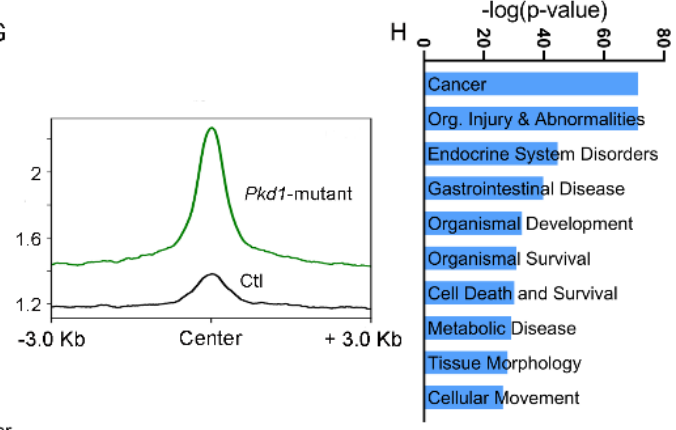

Figure 2. Comparative H3K27ac ChIP-seq uncovering the enhancer landscape in Pkd1mutant kidneys. A. Graphic illustration of the comparative H3K27ac ChIP-Seq experimental design is shown. ChIP-seq was performed using three biological replicate samples, each containing pooled chromatin from four 16-day-old kidneys from control or Pkd1-mutant mice. B. Heatmap showing the signal intensity of H3K27ac (+/- 3kb) around the center of each differentially activated enhancer ordered by mean signal. Pkd1-mutant kidneys gained (higher H3K27ac level) 16560 enhancers and lost (lower H3K27ac level) 1552 enhancers. C. Pie chart depicting the genome-wide distribution of the differentially activated enhancers is shown. D. Heatmap shows the comparison of RPKM of differentially activated enhancers between control and Pkd1-mutant kidneys and wildtype E15.5 and P0 kidneys. E. The bulk H3K27Ac ChIP-seq data were deconvoluted using the E18.5 wildtype snATAC-seq dataset. The cellular distribution of activated enhancers is shown. F. Overlap of the Pkd1-mutant ChIP-Seq and RNA-Seq showing that $>90 \%$ of upregulated genes are located in a TAD which co-houses an activated enhancer, whereas 64\% of downregulated genes are located in a TAD which co-houses a lost enhancer. G. Average $\mathrm{H} 3 \mathrm{~K} 27 \mathrm{ac}$ signal intensity of enhancer regions in control (black) and Pkd1-mutant (green) kidneys samples within TADs that houses upregulated genes. H. Ingenuity Pathway Analysis depicting the top pathways regulated by active enhancer upregulated gene pairs. 
A
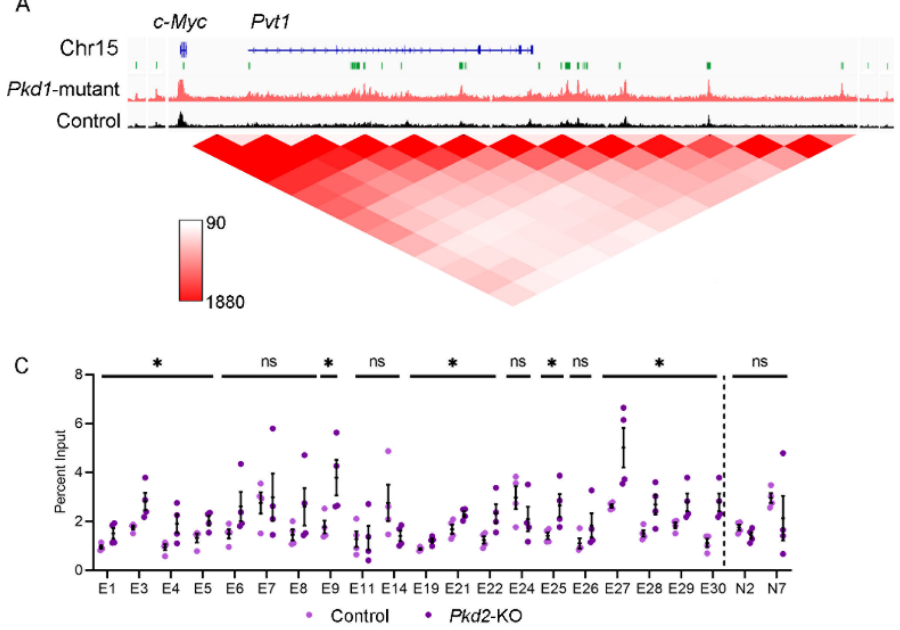
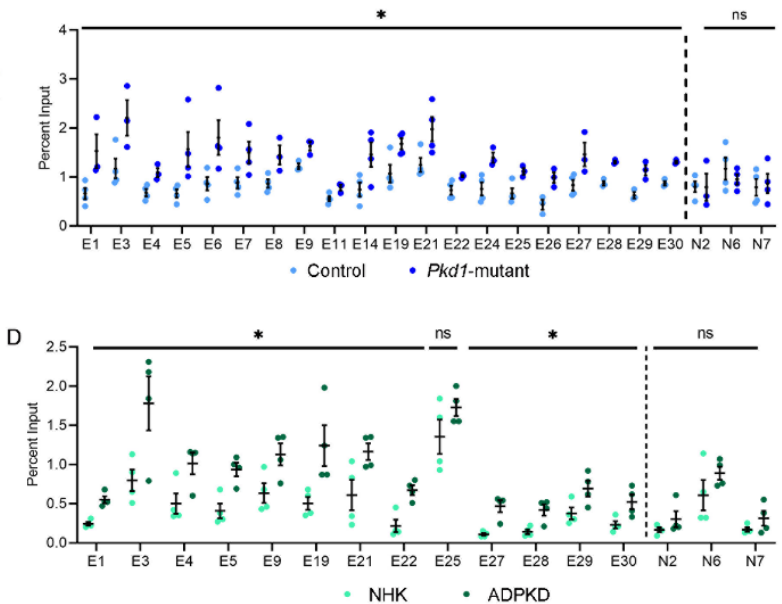

Figure 3. Evolutionarily conserved enhancer cluster in the $c-M y c$ locus is activated in ADPKD models. A. ChIP-seq tracks showing a higher H3K27ac signal within the $c-M y c$ locus in 16-day-old Pkd1-mutant compared to control kidneys. The activated enhancers are marked as green rectangles and denoted as E1-E30 (numbered in $5^{\prime}$ to $3^{\prime}$ orientation). A chromatin contact map (red) for the $c-M y c$ locus derived from $\mathrm{Hi}-\mathrm{C}$ mouse embryonic stem cell dataset is shown. BD. ChIP-qPCR validation of the E1-E30 enhancers in Pkd1-mutant (B), Pkd2-KO (C), and human ADPKD kidneys (D) compared to their respective controls. $N=3-4$ all groups; error bars indicate SEM; * $P<0.05$. ns $P>0.05$; Student's $t$-test. 
A

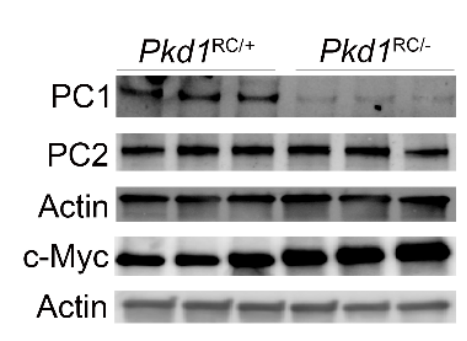

B

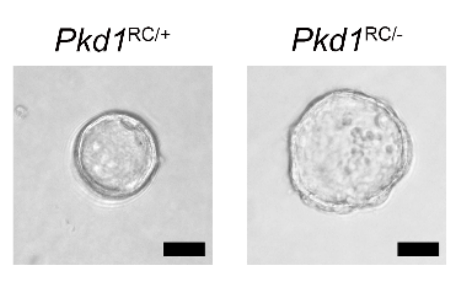

E

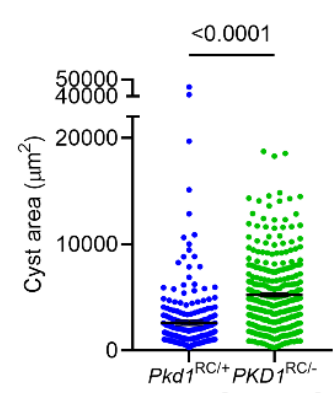

Mean $2600 \mu \mathrm{m}^{2} 5249 \mu \mathrm{m}^{2}$
C

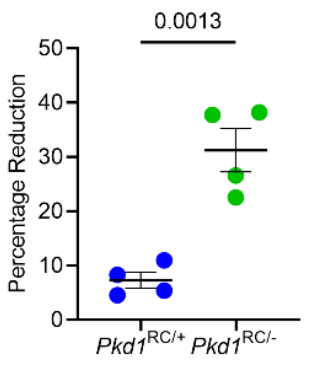

D

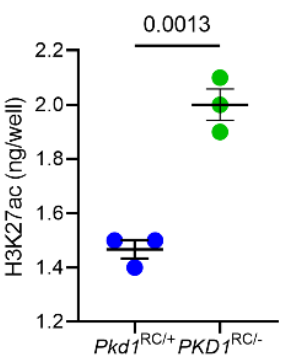

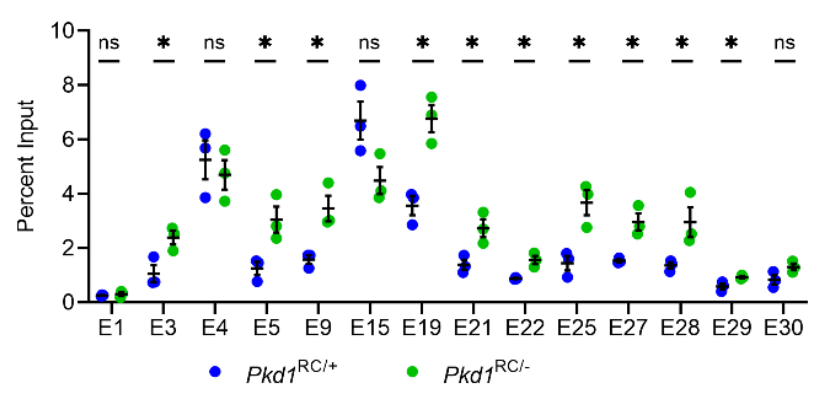

- $P k d 1^{\mathrm{RC} /+}$ - $P k d 1^{\mathrm{RC} /-}$

F

G
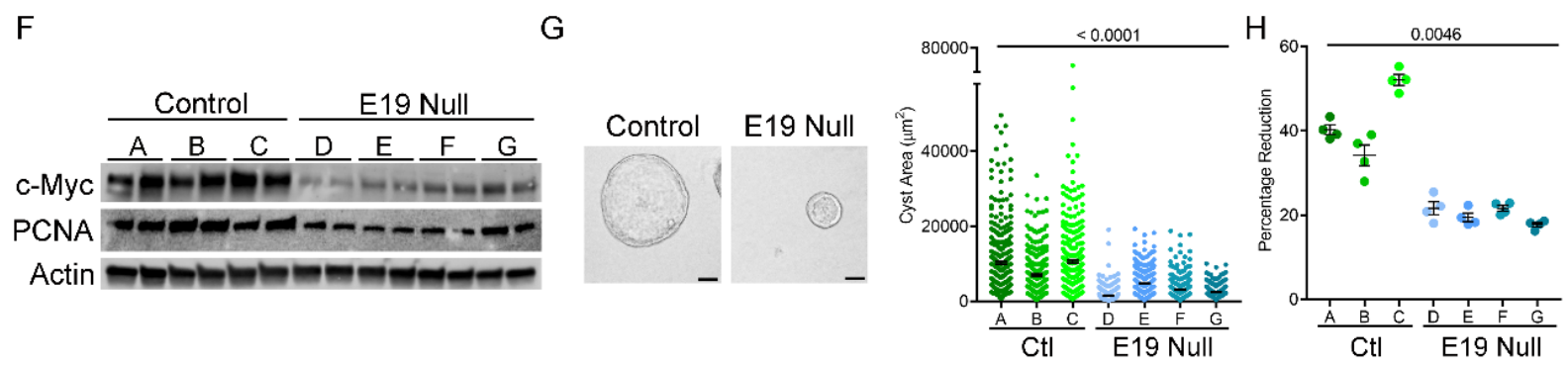

I

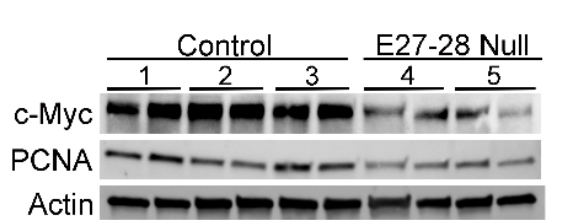

J

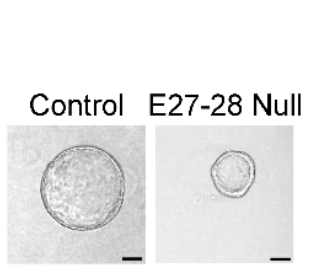

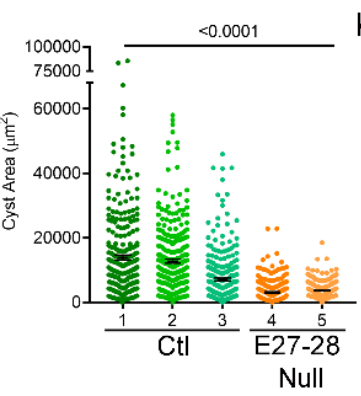

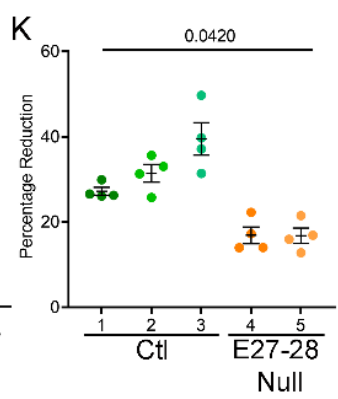

Figure 4. Enhancers activate c-Myc and regulate proliferation and cyst growth in Pkd1mutant cells. A. Western blot analysis showing reduced Polycystin1 (PC1) expression in $P k d 1^{\mathrm{RC} /-}$ cells compared to parental $P k d 1^{\mathrm{RC} /+}$ cells. Polycystin2 (PC2) expression remained unchanged, whereas c-Myc expression was increased in $P k d 1^{\mathrm{RC} /-}$ compared to $P k d 1^{\mathrm{RC} /+}$ cells. Actin serves as the normalizing loading control. B. Representative images and quantification showing increased cyst size of $P k d 1^{\mathrm{RC} /-}$ compared to $P k d 1^{\mathrm{RC} /+}$ cells grown in 3D Matrigel for 7days. C. Alamar Blue measurement 12 hours after incubation showing increased proliferation of $P k d 1^{\mathrm{RC} /-}$ compared to $P k d 1^{\mathrm{RC} /{ }^{+}}$cells. D. ELISA showing higher global H3K27ac levels in $P k d 1^{\mathrm{RC} /-}$ compared to $P k d 1^{R C /+}$ cells. E. Comparative H3K27ac ChIP-qPCR validation of the c-Myc locus enhancers in $P k d 1^{\mathrm{RC} /-}$ and $P k d 1^{\mathrm{RC} /+}$ cells. F. Western blot analysis showing reduced c-Myc expression in $P k d 1^{\mathrm{RC} /-}$ cell lines lacking the $\mathrm{E} 19$ enhancer compared to the unedited $P k d 1^{\mathrm{RC} / \text { - }}$ 
parental cells. G. Representative images and quantification showing reduced cyst size of E19edited compared to unedited $P k d 1^{\mathrm{RC} /-}$ cell lines grown 3D Matrigel for 7-days. H. Alamar Blueassessed proliferation rate of E19-edited and unedited $P k d 1^{\text {RCl- }}$ cells is shown. I-K. Western blot analysis, images and quantification of 3D cyst size, and Alamar Blue measurements showing reduced $\mathrm{c}-$ Myc expression, cyst size, and proliferation of $P k d 1^{\mathrm{RC} /-}$ cells lacking the E27-28 enhancer cluster compared to the unedited parental $P k d 1^{\mathrm{RC} /-}$ cells. Images were taken at $20 \mathrm{x}$ magnification. $\mathrm{N}=300$ (from 3 biological replicates) for all cyst measurements. $\mathrm{N}=4$ biological replicates for all Alamar blue measurements. Error bars indicate SEM. ${ }^{*} P<0.05$. ns $=P>0.05$. Statistical analysis: Students $t$-test (B-E) and nested $t$-test $(\mathrm{G}, \mathrm{H}, \mathrm{J}, \mathrm{K})$. Scale bars represent 25 $\mu \mathrm{M}$. 


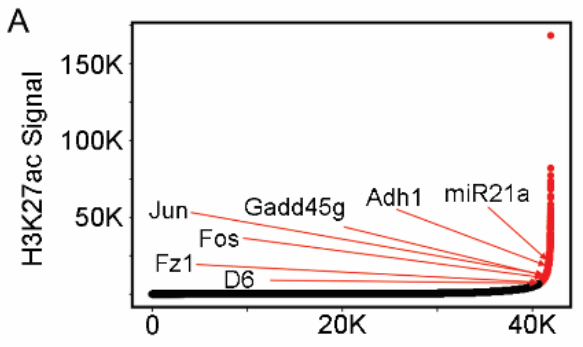

$E$

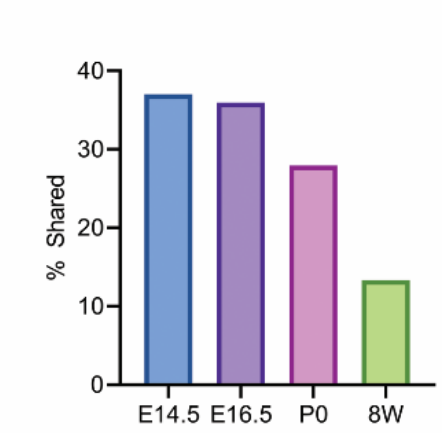

B

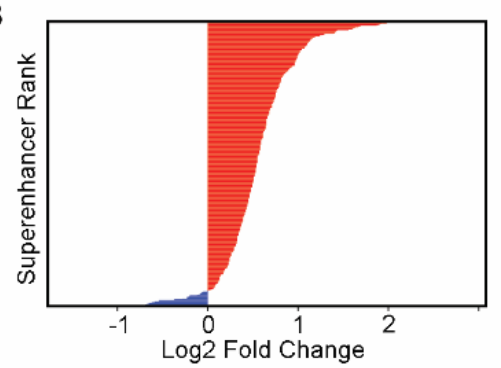

C

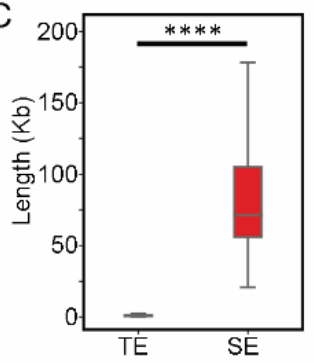

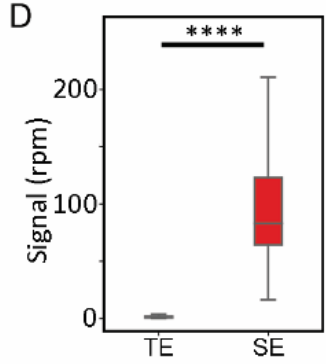

G

$\%$ of superenhancers with motif

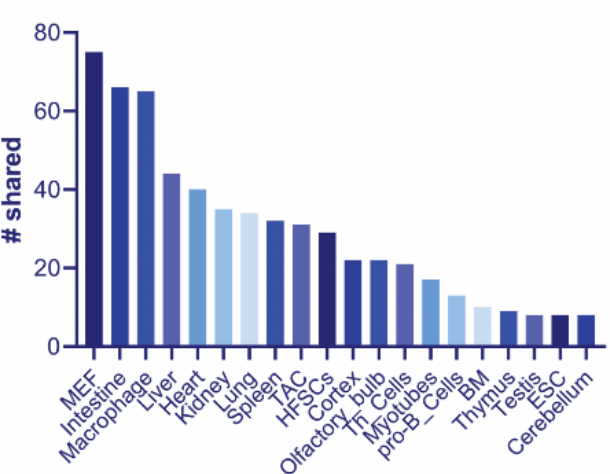

\begin{tabular}{|c|c|c|}
\hline & Name & with motif \\
\hline 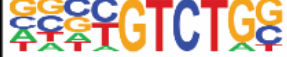 & Smad4 & $20.11 \%$ \\
\hline СTGACTCATC & AP-1 & $10.06 \%$ \\
\hline & EGR1 & $8.05 \%$ \\
\hline & KLF9 & $5.46 \%$ \\
\hline
\end{tabular}
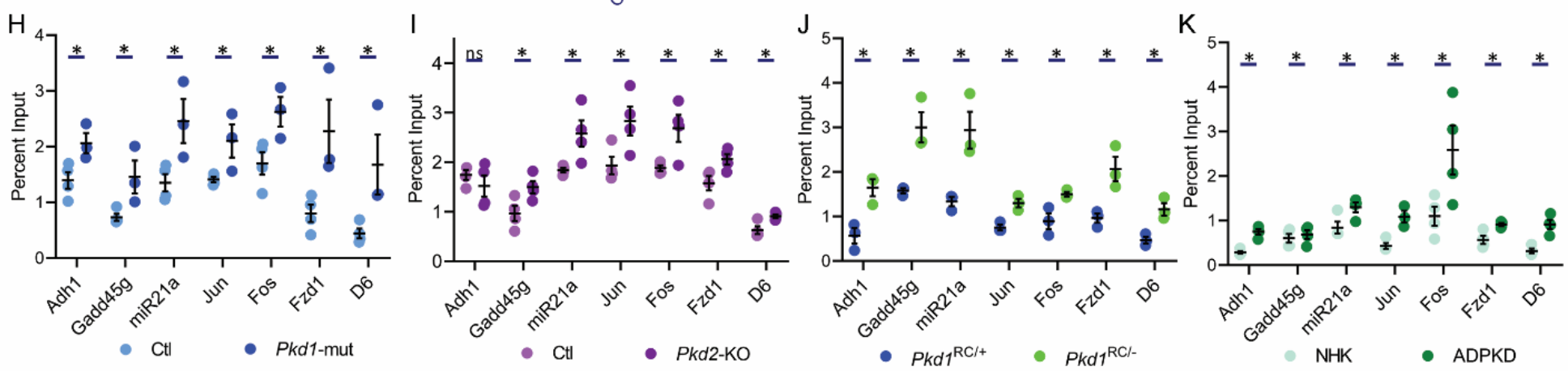

Figure 5. Super-enhancer landscape of ADPKD. A. Hockey stick plot showing rank-ordered, input normalized H3K27ac signal in Pkd1-mutant and control kidneys. The CREs meeting the super-enhancer criteria are highlighted in red. Selected super-enhancers annotated based on nearest neighboring genes are shown. B. The graph depicts all differentially activated superenhancers rank-ordered based on $\log _{2}$ fold change in the H3K27ac signal in 16-day-old Pkd1mutant compared to control kidneys. Red indicates super-enhancers with higher and blue indicates those with lower H3K27ac signal in Pkd1-mutant compared to control kidneys. One hundred five super-enhancers were gained, and five were lost in Pkd1-mutant compared to control kidneys. C and D. Quantification of genomic length and H3K27ac signal intensity of superenhancers (SE) versus total enhancers (TE) is shown. E. Super-enhancers of wildtype E14.5, E16.5, P0, and 8-week (W) kidneys were mapped using the ENCODE data. The graph depicts the level of overlap in the super-enhancer landscape of cystic Pkd1-mutant kidneys and normal developing and adult kidneys. F. The Pkd1-mutant and dbSUPER super-enhancer datasets were cross-compared. The graph depicts the common super-enhancers found in both databases 
bioRxiv preprint doi: https://doi.org/10.1101/2021.11.19.469306; this version posted November 19, 2021. The copyright holder for this preprint (which was not certified by peer review) is the author/funder. All rights reserved. No reuse allowed without permission.

broken down based on cell and tissue type. G. Motif analysis of super-enhancers using the Homer software is shown. H-K. ChIP-qPCR validation of selected super-enhancers in Pkd1-mutant and $P k d 2-K O$ kidneys, $P k d 1^{\text {RC/- }}$ cells, and human ADPKD samples compared to their respective controls. $\mathrm{N}=3-4$ all groups. ${ }^{*} P<0.05,{ }^{* * * *} P<0.001$. ns $=P>0.05$. Error bars indicate SEM. Statistical analysis: Nested $t$-test $(\mathrm{C}$ and $\mathrm{D})$, Student's $t$-test $(\mathrm{H}-\mathrm{K})$ 


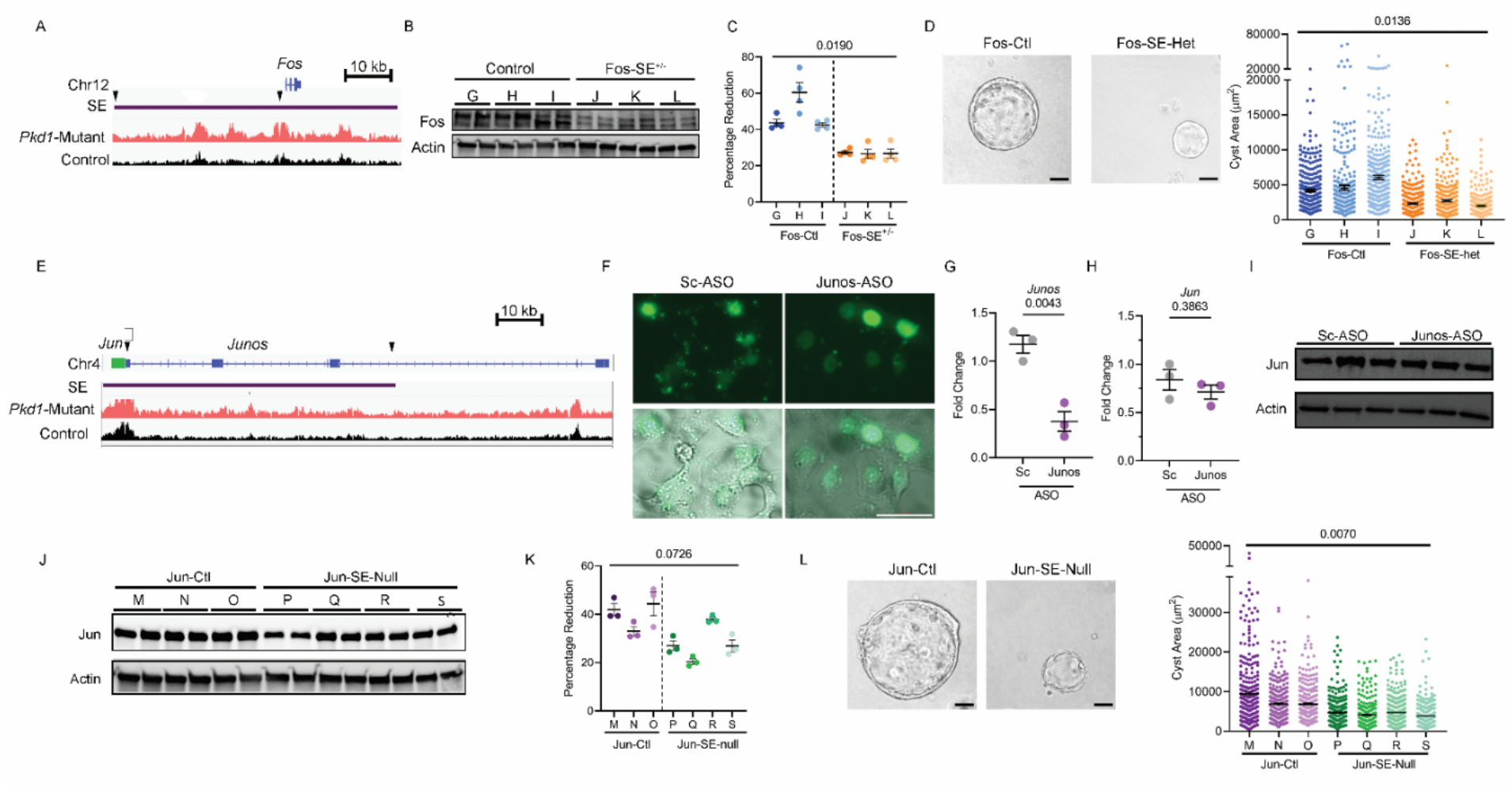

Figure 6. Super-enhancers activate Fos and Jun and regulate proliferation and cyst growth in Pkd1-mutant cells. A. ChIP-seq tracks of the genomic locus encompassing the Fos gene showing higher H3K27ac modification in Pkd1-mutant compared to control kidneys. The Fos super-enhancer is denoted by the purple rectangle. Arrows indicate sites of sgRNAs for CRISPR/Cas9 mediated deletion of the Fos super-enhancer. B. Western blot analysis showing reduced Fos abundance $P k d 1^{R C /-}$ cells with heterozygous deletion of the Fos super-enhancer (Fos-SE ${ }^{+/}$) compared to the unedited parental cell lines (Control). C. Alamar Blue-assessed proliferation of control and $\mathrm{Fos}_{-\mathrm{SE}^{+/}}$cell lines is shown. D. Representative images and quantification showing reduced cyst size in $\mathrm{Fos}_{-} \mathrm{SE}^{+/}$cell lines compared to control cell lines. E. ChIP-seq tracks of the Jun genomic locus showing higher H3K27ac modification in Pkd1-mutant compared to control kidneys. Jun gene is denoted by green rectangle, IncRNA Junos is denoted by blue, and the super-enhancer is denoted by a purple rectangle. Arrows indicate sgRNA targeted sites for super-enhancer deletion. F. Pkd1 $1^{\mathrm{RC} /-}$ cells were transfected with fluorescein amidite (FAM) labeled control antisense oligonucleotide (ASO) or an ASO targeting Junos IncRNA. Fluorescent and bright-field microscopic images showing delivery of control and Junos ASOs into cells 24-hours after transfection. G. qPCR analysis demonstrated reduced Junos transcript abundance in Junos ASO-treated compared to control ASO-treated Pkd1 ${ }^{\mathrm{RC} /-}$ cells. H and I. qPCR and western blot analysis showing that Jun expression was not different in Pkd1 RC/cells treated with control compared to Junos ASO. J. Western blot analysis showing reduced Jun expression in $P k d 1^{\mathrm{RC} /}$ - cell lines lacking the $62 \mathrm{~kb}$ super-enhancer (Jun-SE-null) compared to parental unedited $P k d 1^{\mathrm{RC} /-}$ cells (Jun-ctl). K. Alamar Blue assay demonstrating reduced proliferation in Jun-SE-null compared to Jun-ctl $P k d 1^{\mathrm{RC} /-}$ cells. L. Representative images and quantification showing reduced cyst size in Jun-SE-null compared to Jun-ctl cells. Error bars indicate SEM; Statistical analysis: Nested $t$-test (C, D, K, and L) and Student's $t$-test ( $G$ and $H)$. Scale bars represent $25 \mu \mathrm{M}$ 
A

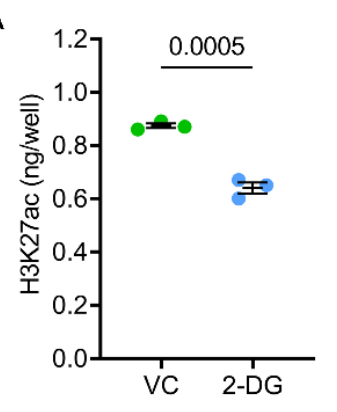

$\mathrm{D}$

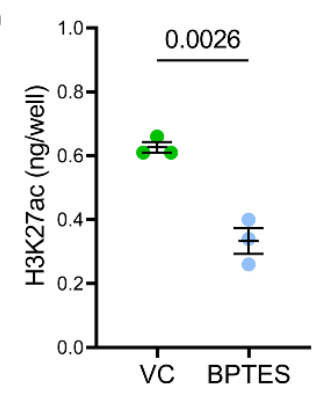

G

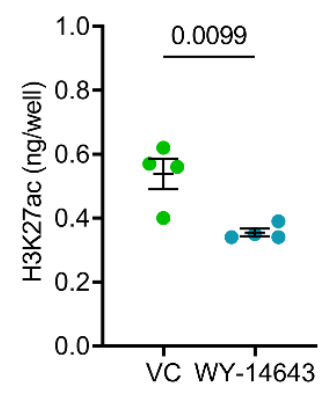

$\mathrm{B}$
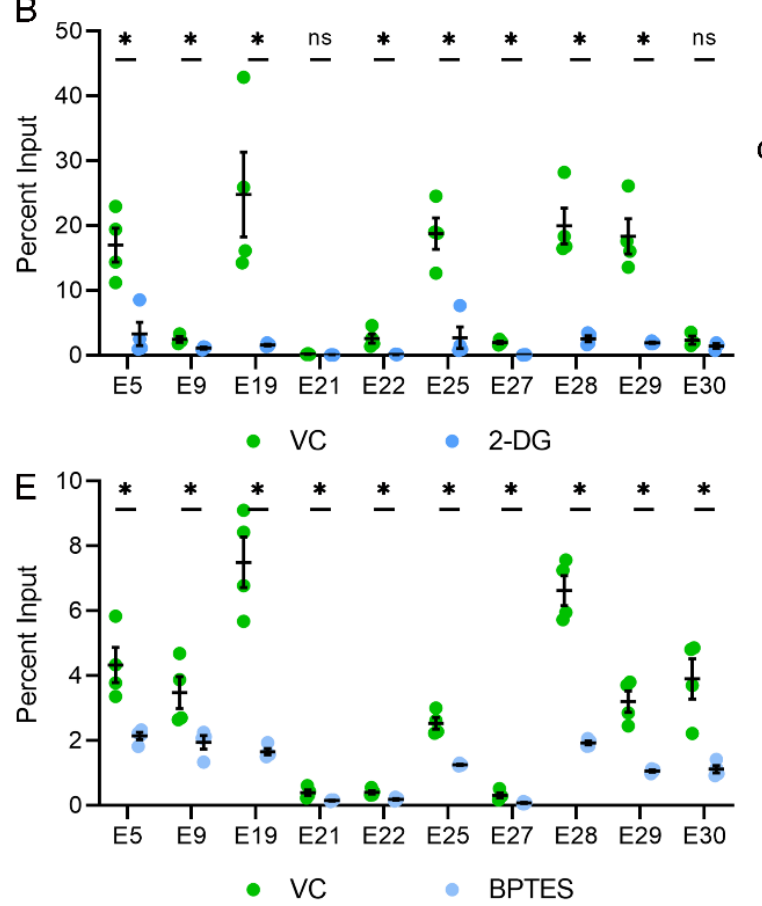

$\mathrm{H}$

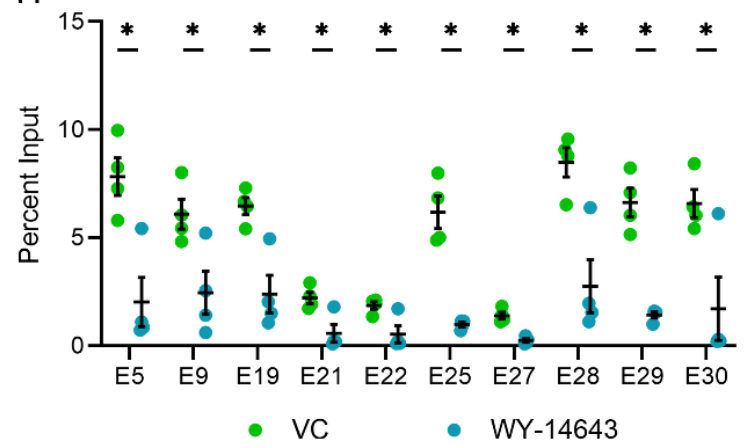

C

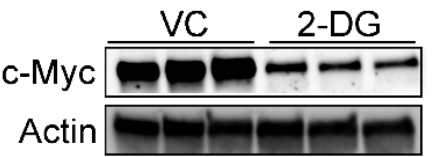

F

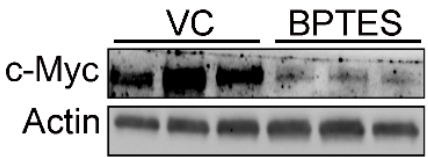

I

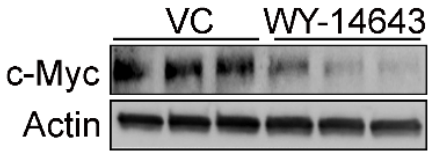

Figure 7. Metabolic pathways influence H3K27ac levels in Pkd1-mutant cells. A. Pkd1 RC/cells were treated with 2-DG, an inhibitor of aerobic glycolysis, or vehicle control for 24 hours. ELISA revealed that histone extracts from 2-DG-treated cells had reduced H3K27ac levels compared to vehicle-treated cells. B. ChIP-qPCR showed reduced H3K27ac modification on $c^{-}$ Myc enhancers in $P k d 1^{\mathrm{RC} /}$ - cells treated with 2-DG compared to vehicle control. C. Western blot analysis showing reduced c-Myc expression in 2-DG-treated compared to vehicle-treated $P k d 1^{\mathrm{RC} /-}$ cells. D. $P k d 1^{\mathrm{RC} /-}$ cells were treated for 48 hours with vehicle or BPTES to inhibit glutaminolysis. ELISA revealed that BPTES-treated cells had a reduced level of $\mathrm{H} 3 \mathrm{~K} 27 \mathrm{ac}$ histone modification compared to vehicle-treated cells. E. ChIP-qPCR showing reduced H3K27ac modification on cMyc locus enhancers in BPTES-treated compared to vehicle-treated Pkd1 ${ }^{\text {RCl- }}$ cells. F. Western blot analysis showing reduced c-Myc expression in Pkd1 ${ }^{\mathrm{RC} /-}$ cells treated with BPTES compared to control vehicle. G-I. Pkd1 ${ }^{\mathrm{RC} /}$ cells were treated for 72 hours with the Ppara agonist WY-4643 or control vehicle. ELISA revealed reduced global H3K27ac levels, ChIP-qPCR showed lower H3K27ac signal on c-Myc enhancers, and Western blot analysis demonstrated c-Myc 
bioRxiv preprint doi: https://doi.org/10.1101/2021.11.19.469306; this version posted November 19, 2021. The copyright holder for this preprint (which was not certified by peer review) is the author/funder. All rights reserved. No reuse allowed without permission.

downregulation in WY-4643-treated compared to vehicle-treated $P k d 1^{\mathrm{RC} /}$ - cells. $\mathrm{N}=3-4$ all groups. ${ }^{*} P<0.05$, ns $=P>0.05$. Error bars indicate SEM. Statistical analysis: Student's $t$-test. 


\section{SUPPLEMENTARY INFORMATION}
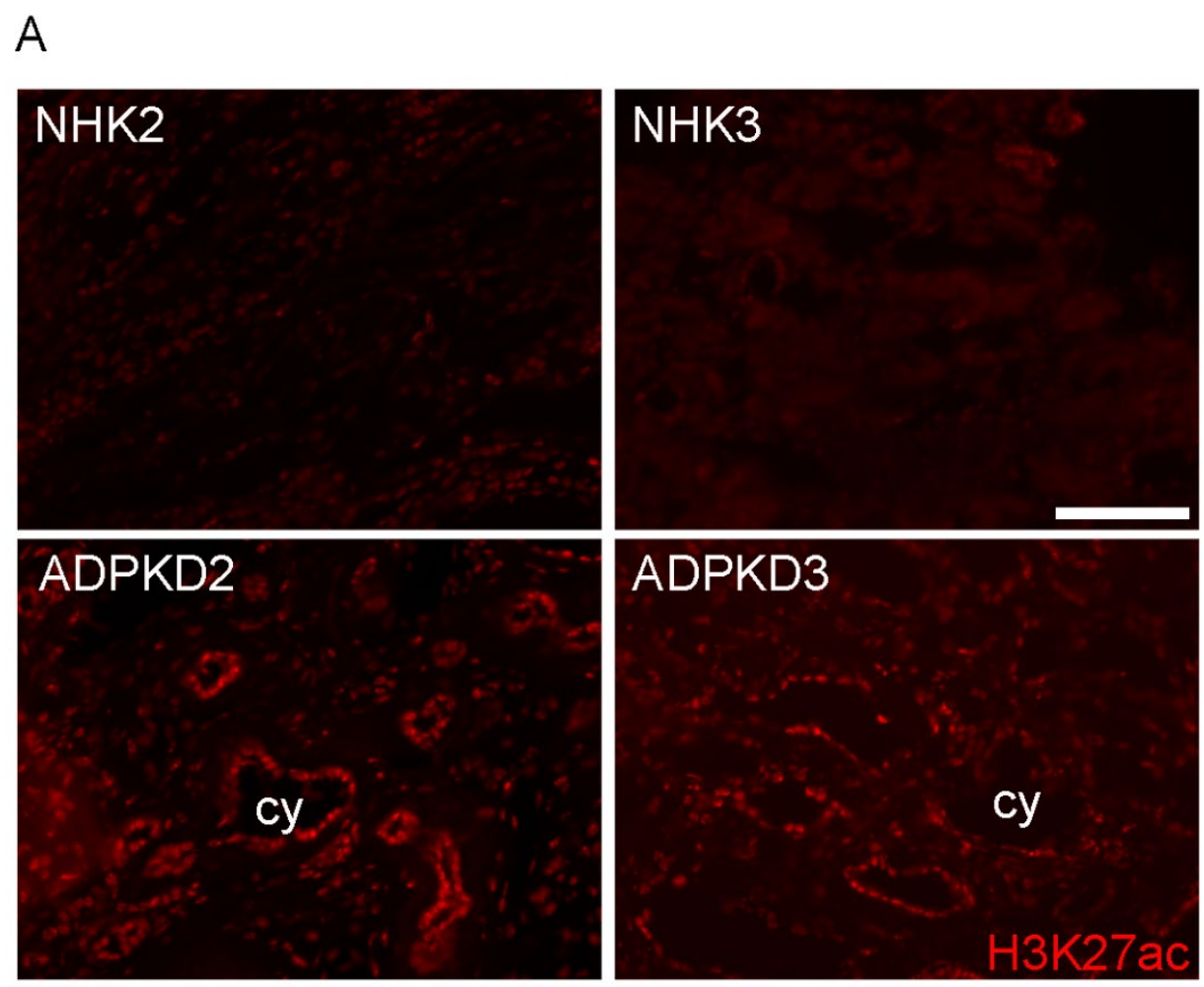

Supplementary Figure 1. Immunofluorescence images showing anti-H3K27ac antibody staining of sections from two normal human kidney (NHK) and human ADPKD samples. Scale bar represents $100 \mu \mathrm{M}$. Cy = cyst 
A

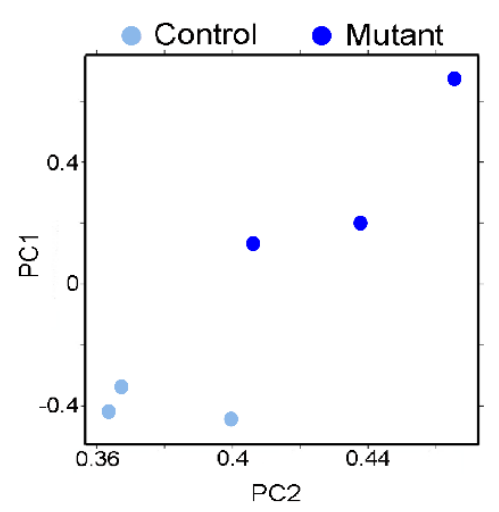

B

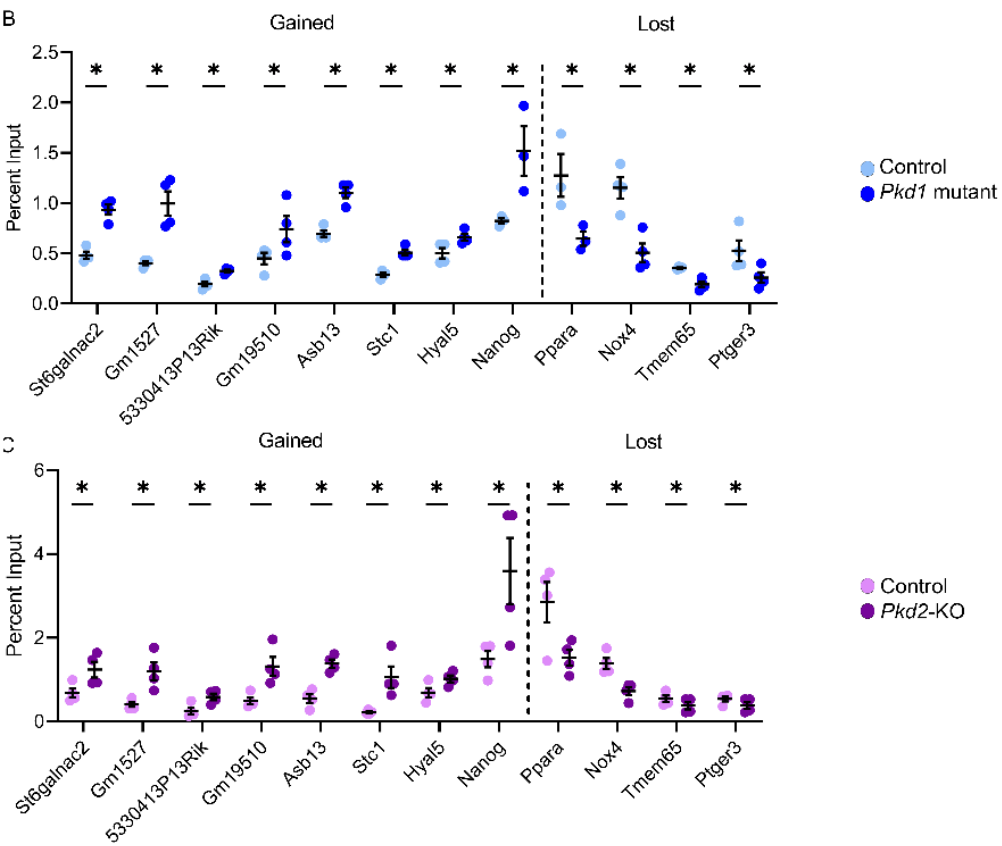

Supplementary Figure 2: A. Principal component analysis of ChIP-Seq data from 16-day-old control and Pkd1-mutant kidneys. B and C. ChIP-qPCR validation of gained and lost enhancers in Pkd1-mutant and $P k d 2-K O$ mice kidneys compared to age-matched littermate controls. $\mathrm{N}=3-4$; ${ }^{*} P<0.05$; error bars represent SEM. Statistical analysis: Student's t-test. 
A

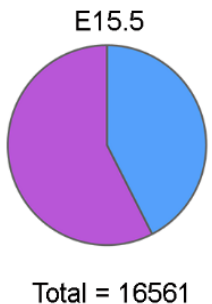

B

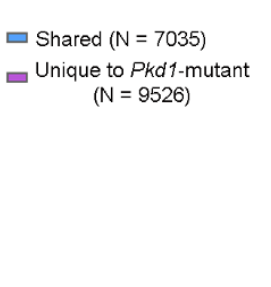

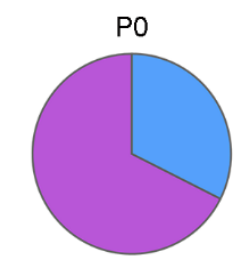

Total $=16561$
C Differentially Activated Enhancers with Expected Chromatin Accessability

- Shared ( $N=5368)$

- Unique to Pkd1-mutant $(N=11193)$

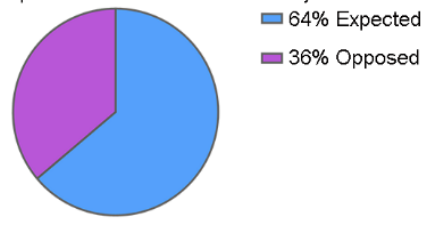

Total $=17598$

Supplementary Figure 3. (A and B) Pie chart demonstrating overlap of the Pkd1-mutant enriched enhancers with those found in E15.5 and P0 kidneys. (C) Pie chart showing the percentage of differentially activated enhancers that fall into areas with expected chromatin accessibility. 


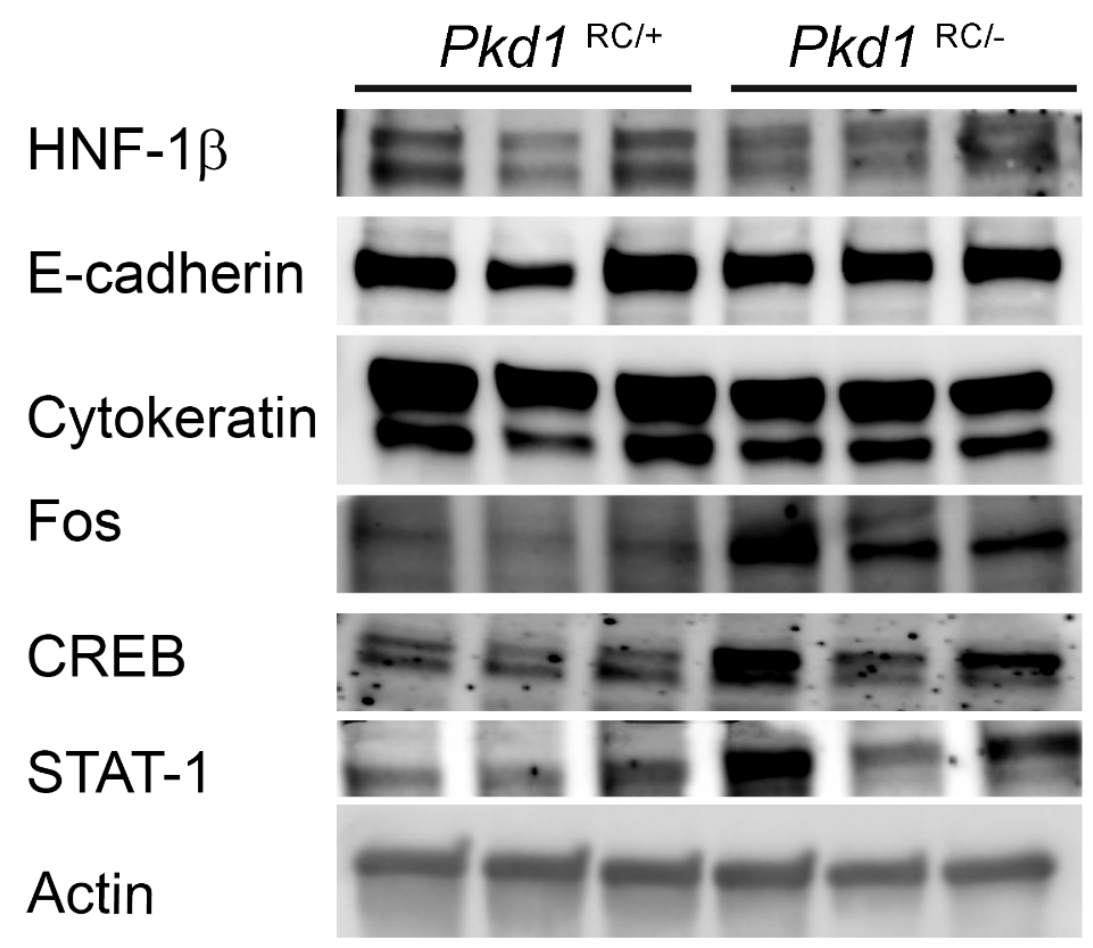

Supplementary Figure 4. Western blot analysis of indicated proteins in $P k d 1^{\mathrm{RC} /+}$ and $P k d 1^{\mathrm{RC} /-}$ cell lines. 
A

$\quad P \frac{C t l}{A B C} \frac{E 19 \text { Null }}{D E F G}$

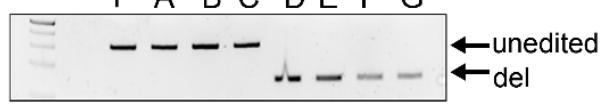

B

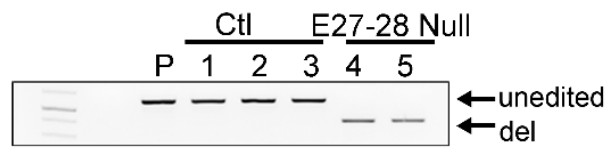

Supplementary Figure 5. A, B. Genotyping confirmation of E19 and E27-28 null cell lines. P $=$ parent control cell line. 


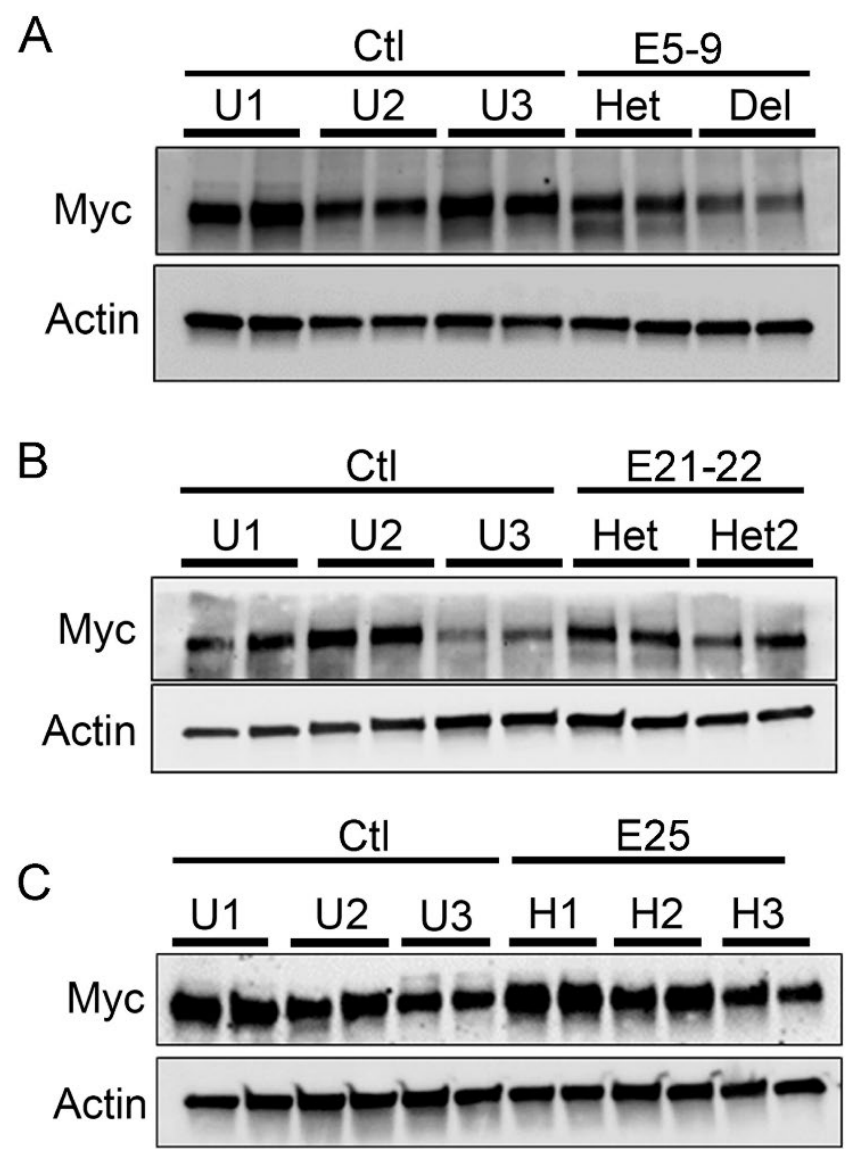

Supplementary Figure 6. A, B and C. Western blot for c-Myc expression for loci E5-9, E21-22, and E25. $\mathrm{P}=$ parent control cell line. $\mathrm{U}=$ unedited cell line, $\mathrm{H}=$ heterozygous cell line Del = homozygous deletion. 


\begin{tabular}{|c|c|c|c|c|c|c|c|c|}
\hline Gene Name & chrom & peak start & peak stop & $\begin{array}{c}\text { peak } \\
\text { width } \\
\text { (bp) }\end{array}$ & $\begin{array}{c}\text { Fold } \\
\text { Change } \\
\text { (Log2) }\end{array}$ & $P$ value & Conserved & $\begin{array}{c}\text { Previously } \\
\text { Reported }\end{array}$ \\
\hline $\mathrm{E} 1$ & chr15 & 60982809 & 60983137 & 329 & 1.58 & 0.0494 & yes & no \\
\hline E2 & chr15 & 61157816 & 61158677 & 862 & 0.96 & 0.0418 & yes & no \\
\hline E3 & chr15 & 61987424 & 61988305 & 882 & 1.59 & 0.00112 & yes & yes \\
\hline $\mathrm{E} 4$ & chr15 & 62039262 & 62039689 & 428 & 1.65 & 0.0303 & yes & yes \\
\hline E5 & chr15 & 62117839 & 62118709 & 871 & 1.64 & 0.00311 & yes & yes \\
\hline E6 & chr15 & 62118807 & 62120422 & 1616 & 2.56 & $5.07 \mathrm{E}-08$ & yes & yes \\
\hline E7 & chr15 & 62120700 & 62121878 & 1179 & 1.94 & 0.00143 & yes & yes \\
\hline E8 & chr15 & 62122325 & 62125270 & 2946 & 2.14 & 0.00000367 & yes & yes \\
\hline E9 & chr15 & 62127781 & 62129102 & 1322 & 2.02 & 0.0000158 & yes & yes \\
\hline $\mathrm{E} 10$ & chr15 & 62129368 & 62129734 & 367 & 1.6 & 0.034 & no & no \\
\hline E11 & chr15 & 62142375 & 62142700 & 326 & 1.63 & 0.032 & yes & yes \\
\hline E12 & chr15 & 62157061 & 62157772 & 712 & 1.69 & 0.0162 & yes & yes \\
\hline E13 & chr15 & 62202823 & 62204455 & 1633 & 1.44 & 0.00158 & no & no \\
\hline E14 & chr15 & 62204551 & 62205624 & 1074 & 1.84 & 0.000596 & yes & no \\
\hline E15 & chr15 & 62207064 & 62207770 & 707 & 1.95 & 0.00795 & no & no \\
\hline E16 & chr15 & 62208443 & 62208807 & 365 & 2.41 & 0.00512 & no & no \\
\hline $\mathrm{E} 17$ & chr15 & 62264530 & 62265748 & 1219 & 1.17 & 0.0422 & no & yes \\
\hline E18 & chr15 & 62281950 & 62282826 & 877 & 1.28 & 0.026 & yes & no \\
\hline E19 & chr15 & 62285294 & 62288292 & 2999 & 1.52 & 0.0000196 & yes & no \\
\hline $\mathrm{E} 20$ & chr15 & 62288424 & 62289166 & 743 & 2.01 & 0.00281 & no & no \\
\hline E21 & chr15 & 62294666 & 62295958 & 1293 & 1.03 & 0.0148 & yes & no \\
\hline E22 & chr15 & 62298809 & 62299565 & 757 & 1.9 & 0.0121 & yes & no \\
\hline E23 & chr15 & 62300495 & 62300964 & 470 & 2.13 & 0.00183 & no & no \\
\hline E24 & chr15 & 62301643 & 62302876 & 1234 & 1.58 & 0.000667 & yes & no \\
\hline E25 & chr15 & 62327111 & 62327978 & 868 & 2.15 & 0.00328 & yes & yes \\
\hline E26 & chr15 & 62395555 & 62398548 & 2994 & 1.2 & 0.00227 & yes & no \\
\hline E27 & chr15 & 62500301 & 62501070 & 770 & 2.92 & 0.00000627 & yes & no \\
\hline E28 & chr15 & 62501314 & 62501898 & 585 & 2.64 & 0.00024 & yes & no \\
\hline E29 & chr15 & 63194175 & 63194585 & 411 & 2.34 & 0.0029 & yes & yes \\
\hline $\mathrm{E} 30$ & chr15 & 63484511 & 63485008 & 498 & 1.6 & 0.0279 & yes & yes \\
\hline
\end{tabular}

Supplementary Table 1. Description of enhancers in the $c-M y c$ locus. 
bioRxiv preprint doi: https://doi.org/10.1101/2021.11.19.469306; this version posted November 19, 2021. The copyright holder for this preprint (which was not certified by peer review) is the author/funder. All rights reserved. No reuse allowed without permission.

\begin{tabular}{|l|l|l|}
\hline \multicolumn{2}{|c|}{ Mouse Primers used for ChIP-qPCR } \\
\hline c-Myc Enhancers & \multicolumn{1}{|c|}{ Forward (5' to $\mathbf{3}^{\prime}$ ) } & \multicolumn{1}{c|}{ Reverse (5' to $\left.\mathbf{3}^{\prime}\right)$} \\
\hline E1 & AGTCTAGACACCTTGAGGCCA & AGAGGGGGCTTCCATTCTG \\
\hline E3 & TCATCTGCGATCCTGACGAC & TGGTAGGAGGCAGCTTCTC \\
\hline E4 & ACCCCACTCTGTTAGGGAGT & CAAGGGAAGCTGGTTATGCA \\
\hline E5 & ACCCAACAAGTGTCCACGTT & CACTTCCACAGGCTTCAGGT \\
\hline E6 & ATCACCAAAGCTGCCATCCA & ACACTGTGGCAGGTGTGAAA \\
\hline E7 & GACTGTGACCTGTACCCAGC & AACTGATCTCGCTGGTGAGG \\
\hline E8 & TGAATGCCCAAACGAGGTGA & TTCACTGAACGATGGGCTCC \\
\hline E9 & AATCCCTGACTGCATTCCTG & GCATTCCAGGCACACCAGTAT \\
\hline E11 & GAGTGTGGACAGCTTAGGGG & GTTCATGCCATCTGTCTTGCT \\
\hline E14 & AGGTTGCATTCCTCTCCGTG & TTCTGTTGACAGCTCCCGTC \\
\hline E19 & CCGTAATGCATTCACAACCA & GGGGAGGAAATGCATGAGTA \\
\hline E20 & CCGCCTCGTGCTTATATTTC & AAAGGAAGGGAAAAGCCAAA \\
\hline E21 & CAATGTCTGCAGGCACAACT & CCCAGCAAGCAAGGTTAAGA \\
\hline E22 & ACCTGTTTTTCCTTAATTCTGCTTGA & GGTGTGGCAGGTGTATCCAA \\
\hline E24 & TCTGCCAGGATCGTTTTCCA & TGGGCTTAACCTGGAACACA \\
\hline E25 & TGTCTTCTGACCTCATGCTTCT & GGTCAAGCTCAGGAAGACGAAA \\
\hline E26 & GTGCCATTTCCTACCCTGCT & TTTCCGAGCGTTGACAACTT \\
\hline E27 & GGGCTACGGCTACAACAGAG & TAAGCAACATTTCCCCAACC \\
\hline E28 & GCCCCTATATTCAGCTCGCT & CACTCCCTTCACAGCGTGAT \\
\hline E29 & TCCTGAACCTTCCCCTTGGT & AGAGGCTGACAAGTTGTGCT \\
\hline E30 & TCCCACCCCCATAATTCTGC & GCCAGCTGCCTTCTAAGAGT \\
\hline Super-enhancers & & \\
\hline D630010B17Rik & TTTGCAGTGTGGATAGCCGT & CCATGCAAGGAAGAGCTGGA \\
\hline Jun & AGCTATCGGGGAGAGGAGAC & CCTTTCCTGACCCTGCCAAT \\
\hline Fos & TGGTCTCTATGGGAACGTCA & ACAGCTGGGAGACAACAACC \\
\hline Adh1 & CCTACCCGCTTAGCTGTCAG & CAATGGACGCTTCTCAAGGG \\
\hline Fzd1 & CTTCCTGCTCTCAGCCAGTC & TGTCACATATGGAGCAGCCT \\
\hline Gadd45g & TAAGAGCAGCCGCACATCAA \\
\hline miR21a & ACAACCAAGGAAACCCTGCA \\
\hline & & \\
\hline
\end{tabular}

Supplementary Table 2. Primer sequences for ChIP-qPCR in mouse kidney samples. 
bioRxiv preprint doi: https://doi.org/10.1101/2021.11.19.469306; this version posted November 19, 2021. The copyright holder for this preprint (which was not certified by peer review) is the author/funder. All rights reserved. No reuse allowed without permission.

\begin{tabular}{|l|l|l|}
\hline \multicolumn{3}{|c|}{ Human Primers used for ChIP-qPCR } \\
\hline \multicolumn{1}{|c|}{ c-Myc Enhancers } & \multicolumn{1}{|c|}{ Forward (5' to $\mathbf{3}^{\prime}$ ) } & \multicolumn{1}{c|}{ Reverse (5' to $\mathbf{3}^{\prime}$ ) } \\
\hline E1 & TTTGGTAGCCCAGACACCTG & CTCACCTTGGGAGTGCTCAG \\
\hline E3 & CGTCCTCGGATTCTCTGCTC & TTCGCTTACCAGAGTCGCTG \\
\hline E4 & GTCTGGGAGAAACCTCGTGG & AATTCCAGTTGGGCCCTTGT \\
\hline E5 & ATCTGATGTCCCCTGCTCCT & AAGCATGAGACCCCAGAAGC \\
\hline E9 & CTCTCTGTGCCTGCATGTTG & TCTGGGCACACCAATATCAA \\
\hline E11 & CTTGAGGGGCTGCTGATAGG & GCGTTCTTGTGTAATCTGTTTGC \\
\hline E19 & AGCATTCGTCTCAGGCACAA & TGGCAAATGAGGTGTGTGACT \\
\hline E21 & CGAGGCCTGATAGATGGAAC & TCACAGTGTTCTGGGTCAGC \\
\hline E22 & CTTGGCATGTCTGGAGAAAGAA & TCTGTGCATTCATACCACTTGT \\
\hline E25 & GGGCAACAGTGGTTTGGGAT & AAACAGCATTGGCAGTCCCT \\
\hline E26 & CGATGTGTGGACCTGAAGCT & GGGGACCGGTGTAGGAAATG \\
\hline E27 & CACCTTTGGGATTGCTTGTT & GCACCTATTATGCAGCGTCA \\
\hline E28 & CCCCTCCCCAATCCTTTGTC & TCCACCTGATCTGCCTCCAT \\
\hline E29 & TTGACTCTTCTCTCCCAACTCC & TATCAGCAGCCAATGCAAAG \\
\hline E30 & CACATTCCTCTCAGCCCATT & GGCAAGAGAAACCAGATCCA \\
\hline Human Super-enhancers & & \\
\hline D630010B17Rik & CTCAAACTGGCCCTGCAGTA & CGGTTCGTTCAGGCTTCTCT \\
\hline Jun & GAGCAGCAGGTCTGTCAAGT & ACCACGTTGCTAGGTGTTGT \\
\hline Fos & CATGGGAACGTCAGGCGG & ACCAAAACAGCAACACAGTCG \\
\hline Adh1 & TACCATGGTGTCAAGCCGAC & GCCACTGAATGCATCAACCC \\
\hline Fzd1 & TGTCATGGAGTTGGCTTGCA & TGCAGTATTCATGGGTGCCC \\
\hline Gadd45g & GGTTTTGTGGGAGACACCA & CGGGACTCCATAGCAACCAG \\
\hline miR21a & TGAATGCTGGTTTAGTCTGGGA & CCGCTGCTGCATTTCGTTAA \\
\hline & & \\
\hline
\end{tabular}

Supplementary Table 3. Primer sequences for ChIP-qPCR in human kidney samples. 
bioRxiv preprint doi: https://doi.org/10.1101/2021.11.19.469306; this version posted November 19, 2021. The copyright holder for this preprint (which was not certified by peer review) is the author/funder. All rights reserved. No reuse allowed without permission.

\begin{tabular}{|l|c|c|}
\hline \multicolumn{1}{|c|}{ Locus } & SgRNA\#1 (5'-3') & SgRNA\#2 (5'-3') \\
\hline c-Myc E1 & GGGAACAGCGATTGGAGGAC & GAAAACAAGAGTAGGGACTA \\
\hline c-Myc E5-E9 & GTCTAAACATTTATCATCCCC & GCTGCTTGCAAATCAACC \\
\hline c-Myc E19 & GAACTTTGTCATGTCAATGC & GCCACTAAAGATTCCAAGCAA \\
\hline c-Myc E21-22 & GCCACTAAAGATTCCAAGCAA & GATATTCAACTAAAGCCAGAG \\
\hline c-Myc E25 & GCACACGGGAGGAACCCAACA & GAGCAGAAAAGTATAACTGAA \\
\hline c-Myc E27-28 & GACCTGTACAGTCAGGATAAT & GTTCTTGGGTCTCTGCTAGGC \\
\hline Jun & GACAGAGGTTTGCTTGGCGA & GTTTACTGGTGAACTTGGTGA \\
\hline Fos & GCTTTGGGAGCTGCCAGAAT & GTTTATCCGTCAGTGTCAAC \\
\hline
\end{tabular}

Supplementary Table 4. Guide RNAs used for CRISPR/Cas9 based deletion of enhancers and super-enhancers 
bioRxiv preprint doi: https://doi.org/10.1101/2021.11.19.469306; this version posted November 19, 2021. The copyright holder for this preprint (which was not certified by peer review) is the author/funder. All rights reserved. No reuse allowed without permission.

\begin{tabular}{|l|l|l|l|}
\hline Enhancer/SE & \multicolumn{1}{|c|}{ Forward Primer (5' to $\left.\mathbf{3}^{\prime}\right)$} & \multicolumn{1}{c|}{ WT_Reverse Primer (5' to $\mathbf{3}$ ) } & \multicolumn{1}{c|}{ Del_Reverse Primer (5' to 3') } \\
\hline c-Myc E19 & AATGGACGGCAATGAACTTTGT & AATGGTTCTTAGAATGGCCGTG & AAGTGAGTGCCTGAGATGAATG \\
\hline c-Myc E27-28 & TCCTACTGCTAAAGAGGCACA & TGTTTGGCAGGTTAAGGACA & CACCAGACCTGCACTAACAAA \\
\hline Jun & CTGCACCCCATTCAACATGTAT & CTGTGGCTACTGGTACTTGGTA & ACCGCTATCTCTAGTTACGCAA \\
\hline Fos & CCAGCAGGGTGAGCAGAC & AAGGGGCTCAGGGGAGATAG & ATGAGACCCGGGTCATTGTC \\
\hline
\end{tabular}

Supplementary Table 5. Primer sequences used to confirm deletion of enhancers and superenhancer loci. 


\section{REFERENCES:}

1 Patel, V., Chowdhury, R. \& Igarashi, P. Advances in the pathogenesis and treatment of polycystic kidney disease. Curr Opin Nephrol Hypertens 18, 99-106, doi:10.1097/MNH.0b013e3283262ab0 [doi] 00041552-200903000-00002 [pii] (2009).

2 Cloutier, M. et al. The societal economic burden of autosomal dominant polycystic kidney disease in the United States. BMC Health Serv Res 20, 126, doi:10.1186/s12913-0204974-4 (2020).

3 Rowe, I. et al. Defective glucose metabolism in polycystic kidney disease identifies a new therapeutic strategy. Nat Med 19, 488-493, doi:10.1038/nm.3092 (2013).

4 Menezes, L. F., Lin, C. C., Zhou, F. \& Germino, G. G. Fatty Acid Oxidation is Impaired in An Orthologous Mouse Model of Autosomal Dominant Polycystic Kidney Disease. EBioMedicine 5, 183-192, doi:10.1016/j.ebiom.2016.01.027 (2016).

5 Lakhia, R. et al. PPARA agonist fenofibrate enhances fatty acid beta-oxidation and attenuates polycystic kidney and liver disease in mice. Am J Physiol Renal Physiol, ajprenal 00352 02017, doi:10.1152/ajprenal.00352.2017 (2017).

6 Flowers, E. M. et al. Lkb1 deficiency confers glutamine dependency in polycystic kidney disease. Nat Commun 9, 814, doi:10.1038/s41467-018-03036-y (2018).

7 Ramalingam, $\mathrm{H}$. et al. A methionine-Mett|3-N(6)-methyladenosine axis promotes polycystic kidney disease. Cell Metab 33, 1234-1247 e1237, doi:10.1016/j.cmet.2021.03.024 (2021).

8 Lakhia, R. et al. Interstitial microRNA miR-214 attenuates inflammation and polycystic kidney disease progression. JCI Insight 5, doi:10.1172/jci.insight.133785 (2020).

9 Torres, V. E. et al. Tolvaptan in Later-Stage Autosomal Dominant Polycystic Kidney Disease. N Engl J Med 377, 1930-1942, doi:10.1056/NEJMoa1710030 (2017).

10 Torres, V. E. et al. Effect of Tolvaptan in Autosomal Dominant Polycystic Kidney Disease by CKD Stage: Results from the TEMPO 3:4 Trial. Clin J Am Soc Nephrol 11, 803-811, doi:10.2215/CJN.06300615 (2016).

11 Lakhia, R. et al. PPARalpha agonist fenofibrate enhances fatty acid beta-oxidation and attenuates polycystic kidney and liver disease in mice. Am J Physiol Renal Physiol 314, F122-F131, doi:10.1152/ajprenal.00352.2017 (2018).

12 Lee, E. C. et al. Discovery and preclinical evaluation of anti-miR-17 oligonucleotide RGLS4326 for the treatment of polycystic kidney disease. Nat Commun 10, 4148, doi:10.1038/s41467-019-11918-y (2019).

13 Yheskel, M., Lakhia, R., Cobo-Stark, P., Flaten, A. \& Patel, V. Anti-microRNA screen uncovers miR-17 family within miR-17 92 cluster as the primary driver of kidney cyst growth. Sci Rep 9, 1920, doi:10.1038/s41598-019-38566-y (2019).

14 Ramalingam, H., Yheskel, M. \& Patel, V. Modulation of polycystic kidney disease by noncoding RNAs. Cell Signal 71, 109548, doi:10.1016/j.cellsig.2020.109548 (2020).

15 Heinz, S., Romanoski, C. E., Benner, C. \& Glass, C. K. The selection and function of cell type-specific enhancers. Nat Rev Mol Cell Biol 16, 144-154, doi:10.1038/nrm3949 (2015).

16 Chapuy, B. et al. Discovery and characterization of super-enhancer-associated dependencies in diffuse large B cell lymphoma. Cancer Cell 24, 777-790, doi:10.1016/j.ccr.2013.11.003 (2013).

17 Loven, J. et al. Selective inhibition of tumor oncogenes by disruption of super-enhancers. Cell 153, 320-334, doi:10.1016/j.cell.2013.03.036 (2013).

18 Hnisz, D. et al. Super-enhancers in the control of cell identity and disease. Cell 155, 934947, doi:10.1016/j.cell.2013.09.053 (2013). 
19 Martinez, M. F. et al. Super-enhancers maintain renin-expressing cell identity and memory to preserve multi-system homeostasis. J Clin Invest 128, 4787-4803, doi:10.1172/JCl121361 (2018).

20 Wilflingseder, J. et al. Enhancer and super-enhancer dynamics in repair after ischemic acute kidney injury. Nat Commun 11, 3383, doi:10.1038/s41467-020-17205-5 (2020).

21 Hajarnis, S. et al. microRNA-17 family promotes polycystic kidney disease progression through modulation of mitochondrial metabolism. Nat Commun 8, 14395, doi:10.1038/ncomms14395 (2017).

22 Le, N. H. et al. Increased activity of activator protein-1 transcription factor components ATF2, c-Jun, and c-Fos in human and mouse autosomal dominant polycystic kidney disease. J Am Soc Nephrol 16, 2724-2731, doi:10.1681/ASN.2004110913 (2005).

23 Wang, Q. et al. Adenylyl cyclase 5 deficiency reduces renal cyclic AMP and cyst growth in an orthologous mouse model of polycystic kidney disease. Kidney Int 93, 403-415, doi:10.1016/j.kint.2017.08.005 (2018).

24 Shao, X., Somlo, S. \& Igarashi, P. Epithelial-specific Cre/lox recombination in the developing kidney and genitourinary tract. J Am Soc Nephrol 13, 1837-1846. (2002).

25 Williams, S. S. et al. Tissue-specific regulation of the mouse Pkhd1 (ARPKD) gene promoter. Am J Physiol Renal Physiol 307, F356-368, doi:10.1152/ajprenal.00422.2013 (2014).

26 Hopp, K. et al. Functional polycystin-1 dosage governs autosomal dominant polycystic kidney disease severity. J Clin Invest 122, 4257-4273, doi:10.1172/JCI64313 (2012).

27 Shibazaki, S. et al. Cyst formation and activation of the extracellular regulated kinase pathway after kidney specific inactivation of Pkd1. Hum Mol Genet (2008).

28 Nishio, S. et al. Loss of oriented cell division does not initiate cyst formation. J Am Soc Nephrol 21, 295-302, doi:ASN.2009060603 [pii] 10.1681/ASN.2009060603 [doi] (2010).

29 Grimm, D. H. et al. Polycystin-1 distribution is modulated by polycystin-2 expression in mammalian cells. J Biol Chem 278, 36786-36793. Epub 32003 Jul 36782. (2003).

$30 \mathrm{Li}, \mathrm{H}$. \& Durbin, R. Fast and accurate long-read alignment with Burrows-Wheeler transform. Bioinformatics 26, 589-595, doi:10.1093/bioinformatics/btp698 (2010).

31 Tarasov, A., Vilella, A. J., Cuppen, E., Nijman, I. J. \& Prins, P. Sambamba: fast processing of NGS alignment formats. Bioinformatics 31, 2032-2034, doi:10.1093/bioinformatics/btv098 (2015).

32 Li, H. et al. The Sequence Alignment/Map format and SAMtools. Bioinformatics 25, 20782079, doi:10.1093/bioinformatics/btp352 (2009).

33 Quinlan, A. R. \& Hall, I. M. BEDTools: a flexible suite of utilities for comparing genomic features. Bioinformatics 26, 841-842, doi:10.1093/bioinformatics/btq033 (2010).

34 Landt, S. G. et al. ChIP-seq guidelines and practices of the ENCODE and modENCODE consortia. Genome Res 22, 1813-1831, doi:10.1101/gr.136184.111 (2012).

35 Feng, J., Liu, T., Qin, B., Zhang, Y. \& Liu, X. S. Identifying ChIP-seq enrichment using MACS. Nat Protoc 7, 1728-1740, doi:10.1038/nprot.2012.101 (2012).

36 Frankish, A. et al. GENCODE reference annotation for the human and mouse genomes. Nucleic acids research 47, D766-D773, doi:10.1093/nar/gky955 (2019).

37 Whyte, W. A. et al. Master transcription factors and mediator establish super-enhancers at key cell identity genes. Cell 153, 307-319, doi:10.1016/j.cell.2013.03.035 (2013).

38 Ramirez, F., Dundar, F., Diehl, S., Gruning, B. A. \& Manke, T. deepTools: a flexible platform for exploring deep-sequencing data. Nucleic acids research 42, W187-191, doi:10.1093/nar/gku365 (2014).

39 Yu, G., Wang, L. G. \& He, Q. Y. ChIPseeker: an R/Bioconductor package for ChIP peak annotation, comparison and visualization. Bioinformatics 31, 2382-2383, doi:10.1093/bioinformatics/btv145 (2015). 
40 Heinz, S. et al. Simple combinations of lineage-determining transcription factors prime cisregulatory elements required for macrophage and B cell identities. Mol Cell 38, 576-589, doi:10.1016/j.molcel.2010.05.004 (2010).

41 Miao, Z. et al. Single cell regulatory landscape of the mouse kidney highlights cellular differentiation programs and disease targets. Nat Commun 12, 2277, doi:10.1038/s41467021-22266-1 (2021).

42 Dixon, J. R. et al. Topological domains in mammalian genomes identified by analysis of chromatin interactions. Nature 485, 376-380, doi:10.1038/nature11082 (2012).

43 Ran, F. A. et al. Genome engineering using the CRISPR-Cas9 system. Nat Protoc 8, 2281-2308, doi:10.1038/nprot.2013.143 (2013).

$44 \mathrm{Wu}, \mathrm{Y}$. et al. Correction of a genetic disease in mouse via use of CRISPR-Cas9. Cell Stem Cell 13, 659-662, doi:10.1016/j.stem.2013.10.016 (2013).

45 Kharchenko, P. V. et al. Comprehensive analysis of the chromatin landscape in Drosophila melanogaster. Nature 471, 480-485, doi:10.1038/nature09725 (2011).

46 Piontek, K., Menezes, L. F., Garcia-Gonzalez, M. A., Huso, D. L. \& Germino, G. G. A critical developmental switch defines the kinetics of kidney cyst formation after loss of Pkd1. Nat Med 13, 1490-1495, doi:nm1675 [pii] 10.1038/nm1675 [doi] (2007).

47 Consortium, E. P. An integrated encyclopedia of DNA elements in the human genome. Nature 489, 57-74, doi:10.1038/nature11247 (2012).

48 Davis, C. A. et al. The Encyclopedia of DNA elements (ENCODE): data portal update. Nucleic acids research 46, D794-D801, doi:10.1093/nar/gkx1081 (2018).

49 Trudel, M., Barisoni, L., Lanoix, J. \& D'Agati, V. Polycystic kidney disease in SBM transgenic mice: role of c-myc in disease induction and progression. Am J Path 152, 219229 (1998).

50 Trudel, M., D'Agati, V. \& Costantini, F. c-myc as an inducer of polycystic kidney disease in transgenic mice. Kidney International 39, 665-671 (1991).

51 Bonev, B. et al. Multiscale 3D Genome Rewiring during Mouse Neural Development. Cell 171, 557-572 e524, doi:10.1016/j.cell.2017.09.043 (2017).

52 Lancho, O. \& Herranz, D. The MYC Enhancer-ome: Long-Range Transcriptional Regulation of MYC in Cancer. Trends Cancer 4, 810-822, doi:10.1016/j.trecan.2018.10.003 (2018).

53 Rossetti, S. et al. Incompletely penetrant PKD1 alleles suggest a role for gene dosage in cyst initiation in polycystic kidney disease. Kidney Int 75, 848-855, doi:10.1038/ki.2008.686 (2009).

54 Cai, L., Sutter, B. M., Li, B. \& Tu, B. P. Acetyl-CoA induces cell growth and proliferation by promoting the acetylation of histones at growth genes. Mol Cell 42, 426-437, doi:10.1016/j.molcel.2011.05.004 (2011).

55 Wellen, K. E. et al. ATP-citrate lyase links cellular metabolism to histone acetylation. Science 324, 1076-1080, doi:10.1126/science.1164097 (2009).

56 Lu, C. \& Thompson, C. B. Metabolic regulation of epigenetics. Cell Metab 16, 9-17, doi:10.1016/j.cmet.2012.06.001 (2012).

$57 \mathrm{Mi}$, Z. et al. Super-enhancer-driven metabolic reprogramming promotes cystogenesis in autosomal dominant polycystic kidney disease. Nat Metab 2, 717-731, doi:10.1038/s42255-020-0227-4 (2020).

58 Gazon, H., Barbeau, B., Mesnard, J. M. \& Peloponese, J. M., Jr. Hijacking of the AP-1 Signaling Pathway during Development of ATL. Front Microbiol 8, 2686, doi:10.3389/fmicb.2017.02686 (2017).

59 Angel, P. \& Karin, M. The role of Jun, Fos and the AP-1 complex in cell-proliferation and transformation. Biochim Biophys Acta 1072, 129-157, doi:10.1016/0304-419x(91)900119 (1991). 
60 Bahr, C. et al. A Myc enhancer cluster regulates normal and leukaemic haematopoietic stem cell hierarchies. Nature 553, 515-520, doi:10.1038/nature25193 (2018).

61 Joo, J. Y., Schaukowitch, K., Farbiak, L., Kilaru, G. \& Kim, T. K. Stimulus-specific combinatorial functionality of neuronal c-fos enhancers. Nat Neurosci 19, 75-83, doi:10.1038/nn.4170 (2016).

62 Santiago-Algarra, D., Dao, L. T. M., Pradel, L., Espana, A. \& Spicuglia, S. Recent advances in high-throughput approaches to dissect enhancer function. F1000Res 6, 939, doi:10.12688/f1000research.11581.1 (2017). 